\title{
CONNECTED HOPF ALGEBRAS OF GELFAND-KIRILLOV DIMENSION FOUR
}

\author{
D.-G. WANG, J. J. ZHANG, AND G. ZHUANG
}

Abstract. We classify connected Hopf algebras of Gelfand-Kirillov dimension 4 over an algebraically closed field of characteristic zero.

\section{INTRODUCTION}

For the introduction and most of the paper we assume that the base field $k$ is algebraically closed of characteristic zero. Noncommutative Hopf algebras of finite Gelfand-Kirillov dimension (GK-dimension, for short) have been studied in several papers; see for example [AA, AS1, AS2, BZ, GZ2, Li, WZZ1, WZZ2, Zh1, Zh2, . The third-named author proved that if a Hopf algebra $H$ is connected, then the associated graded Hopf algebra gr $H$ with respect to the coradical filtration is isomorphic to a commutative Hopf algebra [Zh2, Proposition 6.4]. If $H$ has finite GK-dimension, then gr $H$ is isomorphic to the polynomial ring $k\left[x_{1}, \cdots, x_{n}\right]$ [Zh2, Theorem 6.10], namely, the regular functions $\mathcal{O}(G)$ on a unipotent group $G$, or equivalently, the graded dual $U(\mathfrak{L})^{*}$ of the universal enveloping algebra over a graded Lie algebra $\mathfrak{L}$, which is called the lantern of $H$. Since the coradical filtration is naturally associated to the given Hopf algebra, the lantern $\mathfrak{L}$, as well as the associated unipotent group $G$, are invariants of $H$. This observation motivates the following two related questions.

Question 0.1. What are the invariants of $H$ that determine completely the Hopf algebra structure of $H$ ?

Question 0.2. Can we classify all connected Hopf algebras of finite GK-dimension?

The first question was suggested by Andruskiewitsch and Brown. Also see the talk given by Brown at the Banff workshop [Br3]. The second question is a subquestion of several motivating questions for a couple of ongoing classification projects initiated by many people such as Andruskiewitsch, Schneider, Brown, Goodearl and their collaborators. Some studies of general Hopf algebras of GK-dimension 1 and 2 are given in $[\mathrm{BZ}, \mathrm{Li}, \mathrm{GZ2}, \mathrm{WZZ2}$ by using homological tools. There is little chance to list all isomorphism classes of connected Hopf algebras. A more practical question is along the lines of Question 0.1 can we classify connected Hopf algebras in terms of their invariants?

The connected hypothesis is quite restrictive, but there are some interesting new Hopf algebras in this class even when the GK-dimension is 3; see the classification

\footnotetext{
Received by the editors March 29, 2013 and, in revised form, June 13, 2013.

2010 Mathematics Subject Classification. Primary 16T05, 16P90, 16 E10.

Key words and phrases. Universal enveloping algebra, Hopf algebra, Gelfand-Kirillov dimension, coassociative Lie algebra.
} 
of connected Hopf algebras of GK-dimension 3 in [Zh2, Theorem 1.3]. Since $H$ is a deformation of gr $H$, it is possible to understand all $H$ in Question 0.2 if the (Hopf)cohomologies of gr $H$ can be worked out completely. But we will not consider this in the present paper.

The first goal of the paper is to provide some invariants of connected Hopf algebras that help us to understand partially the structure of the Hopf algebra. One of such is the coassociative Lie algebra that was introduced in WZZ3. Our second goal is to study and classify all connected Hopf algebras of GK-dimension 4, which should give us a better sense of how connected Hopf algebras of higher GK-dimension look. Here is the main result.

Theorem 0.3. Let $H$ be a connected Hopf algebra of GK-dimension 4. Then $H$ is isomorphic to one of the following:

(a) Enveloping algebra $U(\mathfrak{g})$ over a Lie algebra $\mathfrak{g}$ of dimension 4.

(b) Enveloping algebra $U(L)$ over an anti-cocommutative coassociative Lie algebra $L$ of dimension 4.

(c) Primitively-thin Hopf algebras of GK-dimension 4.

Here are some details about Theorem 0.3 .

Remark 0.4. Let $H$ be as in Theorem 0.3 and let parts (a), (b), (c) here match up with those of Theorem 0.3 .

(a) All 4-dimensional Lie algebras over the complex numbers $\mathbb{C}$ are listed in the book OV, Theorem 1.1(iv), page 209]. In this case, the lantern $\mathfrak{L}(H)$ is the unique graded Lie algebra of dimension 4 generated by four elements in degree 1, namely, the abelian Lie algebra of dimension 4.

(b) Anti-cocommutative coassociative Lie algebras of dimension 4 are classified in Theorem 3.5. Therefore Hopf algebras in Theorem 0.3 (b) are completely described. In this case, $\mathfrak{L}(H)$ is, up to isomorphism, the unique graded Lie algebra of dimension 4 generated by three elements in degree 1, namely, the Lie algebra $\mathfrak{h}_{3} \oplus k$ where $\mathfrak{h}_{3}$ is the 3-dimensional Heisenberg Lie algebra.

(c) There are exactly four families of primitively-thin Hopf algebras of GKdimension 4, each of which is constructed explicitly in Section 4 see Theorem 4.23. In this case, $\mathfrak{L}(H)$ is, up to isomorphism, the unique graded Lie algebra of dimension 4 generated by two elements in degree 1 .

Some ideas can be extended to connected Hopf algebras of GK-dimension 5. For example, the classification of the lantern of $H$ is given in Remark 2.10. In higher GK-dimension, we have the following result. Let $p(H)$ denote the dimension of the space of all primitive elements. Let $P_{2}(H)$ be the space spanned by all anti-cocommutative elements of $H$.

Theorem 0.5. Suppose $H$ is a connected Hopf algebra. If $p(H)=\operatorname{GKdim} H-1<$ $\infty$, then $H$ is isomorphic to the enveloping algebra over an anti-cocommutative coassociative Lie algebra $P_{2}(H)$. In this case $H$ is completely determined by $P_{2}(H)$.

\section{Preliminaries}

Throughout let $k$ denote a base field. All vector spaces, algebras, coalgebras are over $k$. For any coalgebra $C$, we use $\Delta$ and $\epsilon$ for comultiplication and counit, respectively. We denote the kernel of the counit by $C^{+}$. The coradical $C_{0}$ of $C$ is defined to be the sum of all simple subcoalgebras of $C$. The coalgebra $C$ is called 
pointed if every simple subcoalgebra is 1-dimensional, and is called connected if $C_{0}$ is 1-dimensional. Also, we use $\left\{C_{n}\right\}_{n=0}^{\infty}$ to denote the coradical filtration of $C$ [Mo, 5.2.1].

For a pointed Hopf algebra $H$, the coradical filtration $\left\{H_{n}\right\}_{n=0}^{\infty}$ is a Hopf algebra filtration [Mo, p. 62]. As a consequence, the associated graded algebra is also a Hopf algebra, which we denote by gr $H$. Also, we use gr $H(n)$ to denote the $n$-th homogeneous component of gr $H$ (i.e. gr $H(n)=H_{n} / H_{n-1}$ ).

Let LieAlg be the category of Lie algebras and let HopfAlg $g_{c c}$ be the category of connected cocommutative Hopf algebras. Then the first assertion of the following proposition is a consequence of Milnor-Moore-Cartier-Kostant Theorem $\mathrm{Mo}$, Theorem 5.6.5]. The second assertion is a well known fact in ring theory.

Proposition 1.1. Let char $k=0$. Then the assignment $\mathfrak{g} \rightarrow U(\mathfrak{g})$ defines an equivalence between categories LieAlg and HopfAlg ${ }_{c c}$. If $\operatorname{dim} \mathfrak{g}<\infty$, then

$$
\operatorname{GKdim} U(\mathfrak{g})=\operatorname{gldim} U(\mathfrak{g})=\operatorname{dim} \mathfrak{g} .
$$

In some sense, noncocommutative connected Hopf algebras are a generalization of the universal enveloping algebra over a Lie algebra.

Let $H$ be a connected Hopf algebra. By [Zh2, Proposition 6.4 and Theorem 6.6], gr $H$ is a commutative domain. Suppose gr $H$ is locally finite. Then the graded dual $(\operatorname{gr} H)^{*}$ is a Hopf algebra.

Definition 1.2. Let $H$ be a connected Hopf algebra.

(a) Let $P(H)$ be the space of primitive elements in $H$ and let $p(H)$ be the dimension of $P(H)$.

(b) We say $H$ is locally finite if $p(H)<\infty$, or equivalently, $H_{i}$ in the coradical filtration of $H$ is finite dimensional for all $i$.

(c) $H$ is called primitively-thin if $p(H)=2$.

(d) Suppose $H$ is locally finite. The lantern of $H$ is the graded Lie algebra $\mathfrak{L}(H)$ such that $U(\mathfrak{L}(H)) \cong(\operatorname{gr} H)^{*}$. In other words, $\mathfrak{L}(H)=P\left((\operatorname{gr} H)^{*}\right)$.

The following lemma is easy. Part (a) of the following lemma says that $\mathfrak{L}(H)$ is a kind of abelianization of $H$. Parts (e,f) justify calling $H$ primitively-thin when $p(H)=2$.

Lemma 1.3. Let $H$ be a locally finite connected Hopf algebra.

(a) If $H$ is the (universal) enveloping algebra $U(\mathfrak{g})$ for a finite-dimensional Lie algebra $\mathfrak{g}$, then $\mathfrak{L}(H)$ is the abelian Lie algebra of dimension equal to $\operatorname{dim} \mathfrak{g}$.

(b) $\mathfrak{L}(H)$ is a positively graded Lie algebra generated in degree 1 and $\operatorname{dim} \mathfrak{L}(H)=$ GKdim $H$.

(c) $\mathfrak{L}(H)_{1}=(\operatorname{gr} H)_{1}^{*}=\left(H_{1} / k\right)^{*}=P(H)^{*}$.

(d) $p(H)=1$ if and only if $H=k[x]$.

(e) [Zh1, Lemma 5.11] If GKdim $H \geq 2$, then $p(H) \geq 2$.

(f) $H$ is primitively-thin if and only if $\mathfrak{L}(H)$ is a graded Lie algebra generated by two elements in degree 1.

Proof. (a) In this case gr $H=U(A)$ where $A$ is an abelian Lie algebra with $\operatorname{dim} A=$ $\operatorname{dim} \mathfrak{g}$. So gr $H$ is a commutative and cocommutative Hopf algebra and $(\operatorname{gr} H)^{*} \cong$ gr $H$ as Hopf algebras. Consequently, $(\operatorname{gr} H)^{*} \cong U(A)$. Thus $\mathfrak{L}(H) \cong A$.

(b) Since gr $H$ is coradically graded, $(\operatorname{gr} H)^{*}$ is generated in degree 1 as an algebra. Since $(\operatorname{gr} H)^{*}$ is cocommutative [Zh2, Proposition 6.4] and char $k=0$, 
$(\operatorname{gr} H)^{*} \cong U(\mathfrak{L})$ and $\mathfrak{L}$ is a graded Lie algebra generated in degree 1. Finally,

$\operatorname{GKdim} H=\operatorname{GKdim} \operatorname{gr} H=\operatorname{GKdim}(\operatorname{gr} H)^{*}=\operatorname{dim} \mathfrak{L}(H)$.

(c) It follows from Definition 1.2 and part (b).

(d) If $p(H)=1$, then $\mathfrak{L}(H)_{1}$ is 1-dimensional. By part (b), $\mathfrak{L}(H)$ is generated by $\mathfrak{L}(H)_{1}$. Thus $\mathfrak{L}(H)=\mathfrak{L}(H)_{1}$, which is 1 -dimensional.

$(\mathrm{e}, \mathrm{f})$ These follow from part (b) and Definition 1.2.

Given any graded Lie algebra finitely generated in degree 1 , say $\mathfrak{g}, U(\mathfrak{g})$ is a locally finite graded Hopf algebra. Since $U(\mathfrak{g})$ is isomorphic to $\left(U(\mathfrak{g})^{*}\right)^{*}$, the lantern of the Hopf algebra $H=(U(\mathfrak{g}))^{*}$ is $\mathfrak{g}$. Therefore every graded Lie algebra finitely generated in degree 1 appears as the lantern of some connected Hopf algebras.

Lemma 1.4. Let $H$ be a connected Hopf algebra of GK-dimension 4 and let $\mathfrak{L}$ be the lantern of $H$. Then $\mathfrak{L}$ is isomorphic to one of the following:

(a) the abelian Lie algebra of dimension 4 concentrated in degree 1.

(b) the graded Lie algebra of dimension 4 with a basis $\{a, b, c,[a, b]\}$ where $a, b, c$, are in degree 1 and $[a, b]$ is in degree 2 , subject to the relations $[c, \mathfrak{L}]=0=$ $[[a, b], \mathfrak{L}]$. This Lie algebra is isomorphic to the Lie algebra $\mathfrak{h}_{3} \oplus k$ where $\mathfrak{h}_{3}$ is the 3-dimensional Heisenberg Lie algebra.

(c) the graded Lie algebra of dimension 4 with a basis $\{a, b,[a, b],[[a, b], b]\}$ where $a, b$ are in degree $1,[a, b]$ is in degree 2 and $[[a, b], b]$ is in degree 3 and subject to the relations $[[a, b], a]=0=[[[a, b], b], \mathfrak{L}]=0$.

Proof. By Lemma 1.3(b), $\mathfrak{L}=\bigoplus_{i>1} \mathfrak{L}_{i}$ is a graded Lie algebra generated in degree 1 of dimension 4. By Lemma 1.3) (c,e), the degree 1 component $\mathfrak{L}_{1}$ has dimension either 2 or 3 or 4 .

If $\operatorname{dim} \mathfrak{L}_{1}=4$, then $\mathfrak{L}=\mathfrak{L}_{1}$, which must be abelian. This is case (a).

If $\operatorname{dim} \mathfrak{L}_{1}=3$, pick a basis, say $\{a, b, c\}$ of $\mathfrak{L}_{1}$. Then $\operatorname{dim} \mathfrak{L}_{2}=1$ and $\mathfrak{L}_{i}=0$ for all $i>2$. Let $z$ be a basis of $\mathfrak{L}_{2}$. By linear algebra, up to a basis change, $z=[a, b]$ and $c$ is in the center of $\mathfrak{L}$. This is case (b).

If $\operatorname{dim} \mathfrak{L}_{1}=2$, pick a basis, say $\{a, b\}$ of $\mathfrak{L}_{1}$. Then $\operatorname{dim} \mathfrak{L}_{2}=1$ with a basis $[a, b]$, $\operatorname{dim} \mathfrak{L}_{3}=1$ and $\operatorname{dim} \mathfrak{L}_{i}=0$ for all $i>3$. Then $[[a, b], a]$ and $[[a, b], b]$ are linearly dependent. Up to a base change we may assume that $[[a, b], a]=0$. Thus $[[a, b], b]$ is the fourth basis element of $\mathfrak{L}$. This is case (c).

The three different cases in Lemma 1.4 will be a guideline for our classification.

Later in this paper we will use the cohomology of coalgebras as a tool, which we briefly recall here. Let $k \subset C$ be a connected coalgebra. Then $C^{+}$, the kernel of the counit, becomes a coalgebra without counit by setting

$$
\delta(x)=\Delta(x)-(1 \otimes x+x \otimes 1) .
$$

Let $\Omega C$ be the tensor algebra $T C^{+}$with a differential determined by

$$
\partial(x)=\delta(x)
$$

for all $x \in C^{+}$with given degree 1. Extend $\partial$ to the algebra $\Omega(C)$ as a derivation. Since $\delta$ is coassociative, $\partial$ is a differential (i.e. $\left.\partial^{2}=0\right)$ and $(\Omega C, \partial)$ is a dga. We call $(\Omega C, \partial)$ the cobar construction of $C$. The $i$-th coalgebra cohomology of $C$ is defined to be the $i$-th cohomology of $(\Omega C, \partial)$, namely $H^{i}(\Omega C)$, for any integer $i$. 


\section{Coassociative Lie algebras and their enveloping Algebras}

We first recall the definition of coassociative Lie algebras.

Definition 2.1 ([WZZ3, Definition 1.1]). A Lie algebra $(L,[]$,$) together with a$ coproduct $\delta: L \rightarrow L \otimes L$ is called a coassociative Lie algebra (or CLA, for short) if

(a) $(L, \delta)$ is a coalgebra; namely, $\delta$ is coassociative without counit.

(b) $\delta$ and $[$, ] satisfy the following condition in the usual enveloping algebra $U(L)$ of the Lie algebra $L$ :

$(\mathrm{E} 2.1 .1) \delta([a, b])=b_{1} \otimes\left[a, b_{2}\right]+\left[a, b_{1}\right] \otimes b_{2}+\left[a_{1}, b\right] \otimes a_{2}+a_{1} \otimes\left[a_{2}, b\right]+[\delta(a), \delta(b)]$

for all $a, b \in L$. Here $\delta(x)=x_{1} \otimes x_{2}$ following Sweedler with $\Sigma$ omitted.

Let CoLieAlg be the category of coassociative Lie algebras (CLAs). Some basic properties of CLAs can be found in WZZ3. The enveloping algebra of a CLA is defined as follows.

Definition 2.2 ([WZZ3, Definition 1.8]). Let $L$ be a CLA. The enveloping algebra of $L$, denoted by $U(L)$, is defined to be the bialgebra whose algebra structure is equal to the enveloping algebra of the Lie algebra $L$ without $\delta$, namely, $U(L)=$ $k\langle L\rangle /(a b-b a=[a, b], \forall a, b \in L)$, and whose coalgebra structure is determined by

$$
\Delta(a)=a \otimes 1+1 \otimes a+\delta(a), \quad \epsilon(a)=0
$$

for all $a \in L$.

In general $U(L)$ is not a Hopf algebra, and it is a Hopf algebra if and only if $L$ is locally conilpotent WZZ3, Theorem 0.1, Definition 1.10].

Definition 2.3. Let $L_{1}$ and $L_{2}$ be CLAs.

(a) We say $L_{1}$ and $L_{2}$ are quasi-equivalent, denoted by $L_{1} \sim L_{2}$, if $U\left(L_{1}\right)$ is isomorphic to $U\left(L_{2}\right)$ as bialgebras.

(b) A coassociative coalgebra $(L, \delta)$ is called anti-cocommutative if $\tau \delta=-\delta$ where the flip $\tau: L^{\otimes 2} \rightarrow L^{\otimes 2}$ is defined by $\tau(a \otimes b)=b \otimes a$.

For an anti-cocommutative CLA $L$, the enveloping algebra $U(L)$ is a connected Hopf algebra since $L$ is conilpotent by [WZZ3, Lemma 2.8(b)].

In the rest of this section we assume that char $k \neq 2$. Let $H$ be a general Hopf algebra and let $P(H)$ denote the $k$-subspace of $H$ consisting of all primitive elements in $H$. It is well known that $P(H)$ is a Lie algebra. The dimension of $P(H)$ is denoted by $p(H)$.

Let $\delta_{H}: H \rightarrow H^{\otimes 2}$ be the map defined by

$$
\delta_{H}(h)=\Delta(h)-(h \otimes 1+1 \otimes h)
$$

for all $h \in H$.

Definition 2.4. Let $H$ be a Hopf algebra.

(a) An element $f \in H^{\otimes 2}$ is called symmetric if $\tau(f)=f$. An element $f \in H^{\otimes 2}$ is called skew-symmetric if $\tau(f)=-f$.

(b) Define

$$
P_{2}(H)=\left\{x \in H \mid \delta_{H}(x) \text { is skew-symmetric and lies in } P(H)^{\otimes 2}\right\} .
$$

The dimension of $P_{2}(H)$ is denoted by $p_{2}(H)$. 
(c) The anti-cocommutative space of $H$ is the quotient space $P_{2}(H) / P(H)$, denoted by $P_{2}^{\prime}(H)$. The dimension of $P_{2}^{\prime}(H)$ is denoted by $p_{2}^{\prime}(H)$.

Here is a list of basic properties of $P_{2}(H)$. Part (a) justifies Definition 2.4(b,c).

Lemma 2.5. Let $H$ be a Hopf algebra. Let $\delta=\delta_{H}$ and $[]=,[,]_{H}$.

(a) Every anti-cocommutative subcoalgebra of $(H, \delta)$ is contained in $P_{2}(H)$. Consequently, $\left(P_{2}(H), \delta\right)$ is the largest anti-cocommutative subcoalgebra of $(H, \delta)$.

(b) $P_{2}(H)=\left\{x \in H \mid \delta(x) \subset P_{2}(H)^{\otimes 2}, \tau \delta(x)=-\delta(x)\right\}$.

(c) $P_{2}(H)$ is a Lie subalgebra of $H$ if and only if $[\delta(x), \delta(y)]=0$ for all $x, y \in$ $P_{2}(H)$.

(d) Suppose $P(H)$ is abelian. Then $\left(P_{2}(H),[],\right)$ is a Lie subalgebra of $H$ and $\left[P_{2}(H), P_{2}(H)\right] \subset P(H)$.

(e) If $P_{2}(H) / P(H)$ is 1-dimensional, then $P_{2}(H)$ is a Lie subalgebra of $H$.

(f) $P_{2}(H)$ is a Lie module over $P(H)$.

(g) $p_{2}^{\prime}(H) \leq\left(\begin{array}{c}p(H) \\ 2\end{array}\right)$.

Proof. Clearly $P(H) \subset P_{2}(H)$. By definition, $\delta\left(P_{2}(H)\right) \subset P(H)^{\otimes 2} \subset P_{2}(H)^{\otimes 2}$ and $\tau \delta(x)=-\delta(x)$ for all $x \in P_{2}(H)$. Hence $P_{2}(H)$ is an anti-cocommutative subcoalgebra of $(H, \delta)$.

(a) By definition, $\operatorname{ker} \delta=P(H)$. Let $C$ be any anti-cocommutative subcoalgebra of $(H, \delta)$. By [WZZ3, Lemma 2.8(b)],

$$
\delta(C) \subset(\operatorname{ker} \delta)^{\otimes 2} \subset P(H)^{\otimes 2} .
$$

Since $C$ is anti-cocommutative, $C \subset P_{2}(H)$ by definition.

(b) Let $C=\left\{x \in H \mid \delta(x) \subset P_{2}(H)^{\otimes 2}, \tau \delta(x)=-\delta(x)\right\}$. Then, by definition, $P_{2}(H) \subset C$. So $\delta(C) \subset C^{\otimes 2}$ and $C$ is an anti-cocommutative subcoalgebra of $(H, \delta)$. The assertion now follows from part (a).

(c) For any $x, y \in H$,

$$
\begin{aligned}
\delta([x, y]) & =\Delta([x, y])-[x, y] \otimes 1-1 \otimes[x, y] \\
& =[\Delta(x), \Delta(y)]-[x, y] \otimes 1-1 \otimes[x, y] \\
& =[\delta(x)+x \otimes 1+1 \otimes x, \delta(y)+y \otimes 1+1 \otimes y]-[x, y] \otimes 1-1 \otimes[x, y] \\
& =w(x, y)+v(x, y),
\end{aligned}
$$

where

$$
w(x, y)=[\delta(x), y \otimes 1+1 \otimes y]+[x \otimes 1+1 \otimes x, \delta(y)]
$$

and

$$
v(x, y)=[\delta(x), \delta(y)]
$$

Now let $x, y \in P_{2}(H)$. By definition, $\delta(x), \delta(y) \in P(H)^{\otimes 2}$. In this case $w(x, y) \in$ $P(H)^{\otimes 2}$. Since $\delta(x)$ and $\delta(y)$ are skew-symmetric, so is $w(x, y)$. But $v(x, y)$ is symmetric. Hence, $\delta([x, y])$ is skew-symmetric if and only if $v(x, y)=0$. The assertion follows.

(d) By part (c), $P_{2}(H)$ is a Lie subalgebra of $(H,[]$,$) . For x, y \in P_{2}(H)$, $\delta([x, y])=w(x, y)+v(x, y)$ where $w(x, y), v(x, y)$ are defined as in the proof of part (c). Since $P(H)$ is abelian, both $w(x, y)$ and $v(x, y)$ are 0 . Hence $\delta([x, y])=0$, and consequently, $[x, y] \in P(H)$. The assertion follows.

(e) Since $P_{2}(H) / P(H)$ is 1-dimensional, $P_{2}(H)=k f \oplus P(H)$ for some $f \in P_{2}(H)$. For any $x, y \in P_{2}(H)$, write $x=a f+x_{0}$ and $y=b f+y_{0}$ for some $a, b \in k$ 
and $x_{0}, y_{0} \in P(H)$. Then $\delta(x)=a \delta(f)$ and $\delta(y)=b \delta(f)$. Hence $[\delta(x), \delta(y)]=$ $[a \delta(f), b \delta(f)]=0$. The assertion follows from part (c).

(f) Let $x \in P_{2}(H)$ and $y \in P(H)$. Then $\delta([x, y])=[\delta(x), 1 \otimes y+y \otimes 1] \in P(H)^{\otimes 2}$ from the proof of part (c). Since $\delta(x)$ is skew-symmetric, so is $[\delta(x), 1 \otimes y+y \otimes 1]$. Hence $[x, y] \in P_{2}(H)$.

(g) This follows from the fact that $\delta$ defines a $k$-linear injective map from $P_{2}(H) / P(H) \rightarrow P(H) \wedge P(H)$.

Lemma 2.6. Let $H$ be a Hopf algebra. Suppose that char $k=0$. In parts (c, d, e), assume that $H$ is a connected Hopf algebra.

(a) $p_{2}(H) \leq \operatorname{GKdim} H$.

(b) Let $\mathfrak{g}$ be a Lie algebra. Then $P_{2}(U(\mathfrak{g}))=P(U(\mathfrak{g}))=\mathfrak{g}$.

(c) Let $U$ be the Hopf subalgebra of $H$ generated by $P(H)$. If $U \neq H$, then $P(H) \neq P_{2}(H)$ and $\operatorname{GKdim} U<\operatorname{GKdim} H$.

(d) $P(H) \cong \operatorname{gr} H(1)=P(\operatorname{gr} H)$.

(e) $P_{2}(H) \cong P_{2}(\operatorname{gr} H)$ and $P_{2}(\operatorname{gr} H) \oplus \operatorname{gr} H(1)^{2}=\operatorname{gr} H(1) \oplus \operatorname{gr} H(2)$.

Proof. (a) The subcoalgebra $P_{2}(H)+k 1$ is connected and counital by WZZ3, Lemma 2.4]. Then the subbialgebra of $H$ generated by $P_{2}(H)+k 1$ is connected, and whence a connected Hopf algebra [Mo, Lemma 5.2.1]. Therefore, after replacing it by the Hopf subalgebra generated by $P_{2}(H)+k 1$, we may assume that $H$ is a connected Hopf algebra.

By [Zh2, Theorem 6.10], gr $H \cong k\left[x_{1}, \cdots, x_{n}\right]$ where $n=\operatorname{GKdim} H$. Arrange $\left\{x_{i}\right\}$ so that $\operatorname{deg} x_{i}=1$ for all $i=1, \cdots, p_{1}$ and $\operatorname{deg} x_{i}=2$ for all $i=p_{1}+1, \cdots, p_{2}$ and $\operatorname{deg} x_{i}>2$ for all $i>p_{2}$. Then $\left\{x_{1}, \cdots, x_{p_{1}}\right\}$ is a basis of $\operatorname{gr} H(1)=H_{1} / H_{0} \cong$ $P(H)$, and $\left\{x_{p_{1}+1}, \cdots, x_{p_{2}}\right\}$ is a basis of gr $H(2) / \operatorname{gr} H(1)^{2}$. Since $\operatorname{gr} H(2)=H_{2} / H_{1}$ and $\operatorname{gr} H(1)=H_{1} / H_{0}$, gr $H(2) / \operatorname{gr} H(1)^{2} \cong H_{2} / H_{1}^{2}$. Note that $P_{2}(H)$ is a subspace of $\mathrm{H}_{2}$ by the definition of $P_{2}(H)$. For any $x \in P_{2}(H) \backslash P(H), \delta(x)$ is nonzero and skew-symmetric by the definition of $P_{2}(H)$ and $P(H)$, and for every $y \in H_{1}^{2}$, an easy calculation shows that $\delta(y)$ is symmetric. Let $C$ be a subspace of $P_{2}(H)$ such that $P_{2}(H)=C \oplus P(H)$. Then the above discussion says that $C \subset H_{2}$ and $C \cap H_{1}^{2}=\{0\}$. Therefore

$$
\operatorname{dim} P_{2}(H) / P(H)=\operatorname{dim} C \leq \operatorname{dim} H_{2} / H_{1}^{2}=\operatorname{dim} g r H(2) / \operatorname{gr} H(1)^{2}=p_{2}-p_{1} .
$$

Thus

$p_{2}(H)=\operatorname{dim} P_{2}(H)=\operatorname{dim} C+\operatorname{dim} P(H) \leq\left(p_{2}-p_{1}\right)+p_{1}=p_{2} \leq n=\operatorname{GKdim} H$.

(b) Follows by a direct computation.

(c) Since $U \neq H$, by [Zh2, Lemma 7.4], GKdim $U<\operatorname{GKdim} H$ if $\operatorname{GKdim} U<\infty$.

Since $U$ is the Hopf subalgebra of $H$ generated by $P(H), U$ is cocommutative and, whence, $U$ is the enveloping algebra $U(\mathfrak{g})$ for some Lie algebra $\mathfrak{g}$. Clearly, $\mathfrak{g}=P(U)=P(H)$.

Consider the coradical filtrations $\left\{U_{i}\right\}_{i \in \mathbb{N}}$ and $\left\{H_{i}\right\}_{i \in \mathbb{N}}$ of $U$ and $H$ respectively. Since $U \subsetneq H$, there is a minimal $n$ such that $U_{n} \subsetneq H_{n}$. The equality $P(U)=P(H)$ implies that $n \geq 2$. Pick any $f \in H_{n} \backslash U_{n}, \delta(f) \in H_{n-1}^{\otimes 2}=U_{n-1}^{\otimes 2}$. This means that $\delta(f) \in U^{\otimes 2}$ is a coalgebra 2-cocycle of $U$.

Since $U$ is the enveloping algebra $U(\mathfrak{g}), U$, as a coalgebra, is isomorphic to $U(V)$ where $V$ is the abelian Lie algebra of dimension equal to dim $\mathfrak{g}$. So one can forget about the Lie structure of $\mathfrak{g}$ when computing the coalegbra cohomology of $U$. A cohomology computation shows that any 2-cocycle is congruent to an element in 
$\mathfrak{g} \wedge \mathfrak{g}$ modulo some 2-coboundary. This fact is a special case of a more general result in [WZZ4, Proposition 4.1]. This means that there is an element $g \in U$ such that $\delta(f-g) \in \mathfrak{g} \wedge \mathfrak{g}$. Since $f \notin U, f-g \in H \backslash U$, or $f-g \in P_{2}(H)$ as $\delta(f-g) \in \mathfrak{g} \wedge \mathfrak{g}$ is skew-symmetric. The assertion follows.

(d) By the definition of coradical filtration, $H_{1}=k 1 \oplus P(H)=H_{0} \oplus P(H)$. Hence $P(H) \cong \operatorname{gr} H(1)$. Since gr $H$ is coradically graded [Zh2, Remark 2.2], $P(\operatorname{gr} H)=$ gr $H(1)$.

(e) Let $x \in P_{2}(H)$ and let $a$ be the associated element in gr $H$. If $x \in P(H)$, then $a \in P(\operatorname{gr} H)$ by part (d) and the map $x \rightarrow a$ is an isomorphism when restricted to $P(H)$. Now suppose $x \notin P(H)$. Then $a \notin P(\operatorname{gr} H)$ by part (d). Since $\delta(x) \in$ $P(H)^{\otimes 2}$ is skew-symmetric, so is $\delta(a) \in P(\operatorname{gr} H)^{\otimes 2}$. Thus we have an injective map $P_{2}(H) \rightarrow P_{2}(\operatorname{gr} H)$. Now let $a \in P_{2}(\operatorname{gr} H)$. Since $P(H) \cong \operatorname{gr} H(1)$, we may assume that $a \in \operatorname{gr} H(2)$. Let $\left\{x_{i}\right\}$ be a basis of $P(H)$ and $\left\{y_{i}\right\}$ be the corresponding basis of gr $H(1)$. Then $\delta(a)=\sum_{i, j} c_{i j}\left(y_{i} \otimes y_{j}-y_{j} \otimes y_{i}\right)$ for some $c_{i j} \in k$. Let $x \in H_{2}^{+}$be a preimage of $a$. Then $\delta(x)=\sum_{i, j} c_{i j}\left(x_{i} \otimes x_{j}-x_{j} \otimes x_{i}\right)+1 \otimes z_{1}+z_{2} \otimes 1+c \otimes 1$ where $z_{1}, z_{2} \in P(H)$ and $c \in k$. The counit axiom for $\Delta$ implies that $z_{1}=z_{2}=c=0$. Therefore $x \in P_{2}(H)$. The first assertion follows.

For the second assertion we note that

$$
\operatorname{gr} H(1)=P(\operatorname{gr} H) \subset P_{2}(\operatorname{gr} H) \subset \operatorname{gr} H(1) \oplus \operatorname{gr} H(2)
$$

and that

$$
P_{2}(\operatorname{gr} H) \cap \operatorname{gr} H(1)^{2}=\{0\} .
$$

It remains to show that gr $H(2) \subset P_{2}(\operatorname{gr} H) \oplus \operatorname{gr} H(1)^{2}$. By replacing $H$ with gr $H$, we may assume that $H$ is coradically graded. For any $x \in H_{2}^{+}, \delta(x)=\sum_{i, j} c_{i j} x_{i} \otimes$ $x_{j}+1 \otimes z_{1}+z_{2} \otimes 1+c \otimes 1$ where $x_{i}, z_{1}, z_{2} \in P(H)$ and $c \in k$. The counit axiom for $\Delta$ implies that $z_{1}=z_{2}=c=0$. Replacing $x$ by $x-\sum_{i} \frac{1}{2} c_{i i} x_{i}^{2}-\frac{1}{2} \sum_{i<j}\left(c_{i j}+c_{j i}\right) x_{i} x_{j}$, $\delta(x)$ becomes skew-symmetric. So $x \in P_{2}(H)$. The second assertion follows.

Here is our main result of this section.

Theorem 2.7. Suppose that char $k=0$ and $H$ is a connected Hopf algebra. If

$$
\operatorname{GKdim} H \leq p(H)+1<\infty,
$$

then $H \cong U(L)$ for some anti-cocommutative $C L A L=P_{2}(H)$. If, further, GKdim $H=p(H)$, then $H=U(\mathfrak{g})$ where $\mathfrak{g}=P(H)$.

Proof. Let $\mathfrak{g}$ be the Lie algebra $P(H)$ of the primitive elements of $H$. If $H=U(\mathfrak{g})$, the assertion is trivial as we have a natural embedding LieAlg $\rightarrow$ CoLieAlg.

For the rest of the proof we assume that $H \neq U(\mathfrak{g})$. By Lemma 2.6 (c), $P_{2}(H) \neq$ $P(H)$. Since $P(H)$ is a proper subspace of $P_{2}(H), p(H)+1 \leq p_{2}(H)$. By Lemma 2.5 (h), $p_{2}(H) \leq \mathrm{GKdim} H$. By hypothesis, GKdim $H \leq p(H)+1<\infty$. Therefore there is only one possibility, namely, $p_{2}(H)=p(H)+1=\operatorname{GKdim} H$. By Lemma 2.5(e) $P_{2}(H)$ is a Lie subalgebra of $H$. By Lemma 2.5(a), $\left(P_{2}(H), \delta\right)$ is an anticocommutative subcoalgebra of $(H, \delta)$.

Let $K$ be the subbialgebra of $H$ generated as an algebra by the connected subcoalgebra $\left(P_{2}(H)+k 1, \Delta\right)$. Then $K$ is a Hopf subalgebra of $H$ as mentioned in the proof of Lemma 2.6(a). By Lemma 2.5(a), $P_{2}(K) \supseteq P_{2}(H)$ and clearly $P_{2}(H) \supseteq P_{2}(K)$, so we have $P_{2}(K)=P_{2}(H)$. By Lemma 2.6(b), $P_{2}(U(\mathfrak{g}))=\mathfrak{g}$, 
and by assumption in the previous paragraph and Lemma 2.6 (c), $P_{2}(H) \supsetneq \mathfrak{g}$, so we conclude that $U(\mathfrak{g})$ is a proper subalgebra of $K$. By [Zh2, Lemma 7.4],

$$
\operatorname{GKdim} K \geq \operatorname{GKdim} U(\mathfrak{g})+1=p(H)+1 .
$$

By hypothesis, GKdim $H \leq p(H)+1$ and obviously GKdim $H \geq \operatorname{GKdim} K$. Hence $\operatorname{GKdim} H=\operatorname{GKdim} K=p(H)+1=p_{2}(H)$. By [Zh2, Lemma 7.4], $H=K$.

Next we show that $L:=P_{2}(H)$ is a CLA. By the second paragraph, $L$ is both a Lie algebra and a coalgebra. It remains to verify (E2.1.1). Choose a basis of $\mathfrak{g}$, say $\left\{x_{i}\right\}$, and an element $z \in L \backslash \mathfrak{g}$. Then $\left\{x_{i}\right\} \cup\{z\}$ is a basis of $L$. It is trivial that (E2.1.1) holds for $(a, b)=(z, z)$ and for $(a, b)=\left(x_{i}, x_{j}\right)$ since $\delta\left(x_{i}\right)=0$. It remains to show (E2.1.1) for $(a, b)=\left(z, x_{i}\right)$ (and by symmetry for $(a, b)=\left(x_{i}, z\right)$ ). Since $\delta\left(x_{i}\right)=0$, we have

$$
\begin{aligned}
\delta\left(\left[z, x_{i}\right]\right) & =\left[\delta(z), x_{i} \otimes 1+1 \otimes x_{i}\right] \\
& =\left[z \otimes 1+1 \otimes z, \delta\left(x_{i}\right)\right]+\left[\delta(z), x_{i} \otimes 1+1 \otimes x_{i}\right]+\left[\delta(z), \delta\left(x_{i}\right)\right],
\end{aligned}
$$

which holds in $H^{\otimes 2}$ and hence holds in $L^{\otimes 2}$. Thus the above holds in $U(L)^{\otimes 2}$, which verifies (E2.1.1).

Let $U(L)$ be the enveloping algebra of the CLA $L$. There is a canonical Hopf algebra map $\phi: U(L) \rightarrow H$ sending $x \in L$ to $x$. By [Mo, Theorem 5.3.1], the map $\phi$ is injective since the restriction of $\phi$ on the space of primitive elements, namely, $\left.\phi\right|_{P(H)}$, is injective. Now by [Zh2, Lemma 7.4] again this is an isomorphism since $\operatorname{GKdim} H=\operatorname{GKdim} U(L)$. Finally by the definition of $P_{2}(H), L$ is anticocommutative.

Proposition 2.8. Suppose char $k=0$. Let $L$ be an anti-cocommutative $C L A$.

(a) $P_{2}(U(L))=L$.

(b) Let $P=\operatorname{ker}\left(\delta: L \rightarrow L^{\otimes 2}\right)$. Then $\operatorname{gr} U(L)=k[P \oplus L / P]$ where elements of $P$ are in degree 1 and that of $L / P$ are in degree 2.

(c) The coradical of $U(L)$ is given by $U(L)_{0}=k 1, U(L)_{1}=P+k 1, U(L)_{2}=$ $(P+k 1)^{2}+L$, and, for $n \geq 3$,

$$
U(L)_{n}=\sum_{i=1}^{n-1} U(L)_{i} \cdot U(L)_{n-i} .
$$

(d) Suppose $L$ is finite dimensional. Then $\mathfrak{L}(U(L)) \cong P^{*} \oplus(L / P)^{*}$ where the Lie algebra structure of $P^{*} \oplus(L / P)^{*}$ is induced by the coalgebra structure of $L$.

Proof. For simplicity, we assume that $\operatorname{dim} L<\infty$ in the following proof. The assertion holds in general, but the proof requires more computation, which we omit here.

(a) By construction and Lemma 2.5(a), $L$ is a subspace of $P_{2}(U(L))$. By Lemma 2.6(a),

$$
\operatorname{dim} P_{2}(U(L)) \leq \operatorname{GKdim} U(L)=\operatorname{dim} L \leq \operatorname{dim} P_{2}(U(L)) .
$$

Hence $L=P_{2}(U(L))$ as $L$ is finite dimensional.

(b) By the proof of Lemma 2.6(a), $k[P \oplus L / P]$ is a Hopf subalgebra of gr $U(L)$. Obviously they have the same GK-dimension. Now the assertion follows from Zh2, Lemma 7.4].

(c) This follows from part (b) and the fact that $\operatorname{gr} U(L)$ is coradically graded. 
(d) By part (b), $H:=\operatorname{gr} U(L)=k[P \oplus L / P]$ where $P \oplus L / P$ is the minimal generating space of $H$. Then $P^{*} \oplus(L / P)^{*}$ is a minimal cogenerating space of the dual $K:=H^{*}=(\operatorname{gr} U(L))^{*}$. Let $\left\{x_{i}\right\}_{i}$ and $\left\{y_{j}\right\}_{j}$ be a basis of $P$ and $L / P$ respectively, and $\left\{x_{i}^{*}\right\}_{i}$ and $\left\{y_{j}^{*}\right\}_{j}$ be the dual basis of $P^{*}$ and $(L / P)^{*}$ respectively. It follows from the definition and the fact that $x_{i}$ and $y_{j}$ are generators that both $x_{i}^{*}$ and $y_{j}^{*}$ are primitive elements. Therefore $P^{*} \oplus(L / P)^{*} \subset \mathfrak{L}(U(L))$. Consequently, $P^{*} \oplus(L / P)^{*}=\mathfrak{L}(U(L))$ as they have the same $k$-dimension. Suppose, for any $s$, $\delta\left(y_{s}\right)=\sum_{i<j} c_{s}^{i j}\left(x_{i} \otimes x_{j}-x_{j} \otimes x_{i}\right)$ for some $c_{s}^{i j} \in k$, and define $c_{s}^{j i}=-c_{s}^{i j}$. Then

$$
\Delta_{H}\left(y_{s}\right)=y_{s} \otimes 1+1 \otimes y_{s}+\sum_{i<j} c_{s}^{i j}\left(x_{i} \otimes x_{j}-x_{j} \otimes x_{i}\right) .
$$

By the $k$-linear pairing $K \times H \rightarrow k$, we have

$$
\begin{aligned}
{\left[x_{i}^{*}, x_{j}^{*}\right]\left(y_{t}\right) } & =\left(x_{i}^{*} x_{j}^{*}-x_{j}^{*} x_{i}^{*}\right)\left(y_{t}\right) \\
& =\left(x_{i}^{*} \otimes x_{j}^{*}-x_{j}^{*} \otimes x_{i}^{*}\right)\left(\Delta_{H}\left(y_{t}\right)\right) \\
& =\left(x_{i}^{*} \otimes x_{j}^{*}-x_{j}^{*} \otimes x_{i}^{*}\right)\left(y_{t} \otimes 1+1 \otimes y_{t}+\sum_{i^{\prime}<j^{\prime}} c_{t}^{i^{\prime} j^{\prime}}\left(x_{i^{\prime}} \otimes x_{j^{\prime}}-x_{j^{\prime}} \otimes x_{i^{\prime}}\right)\right) \\
& =2 c_{t}^{i j}=\sum_{s} c_{s}^{i j}\left(2 y_{s}^{*}\right)\left(y_{t}\right) .
\end{aligned}
$$

Hence $\left[x_{i}^{*}, x_{j}^{*}\right]=\sum_{s} 2 c_{s}^{i j} y_{s}^{*}$ for all $i, j$. Therefore the assertion follows since the coefficients $c_{s}^{i j}$ are determined by the coalgebra of $L$.

Proposition 2.9. Let $L$ be a conilpotent $C L A$ of dimension $\leq 4$. Then it is quasiequivalent to an anti-cocommutative CLA.

Proof. By WZZ3, Lemma 2.4], $U(L)$ is a connected Hopf algebra. By Lemma 1.3(e), $P(U(L))$ has dimension at least 2. If $\operatorname{dim} L \leq 3$, the assertion follows from Theorem 2.7 For the rest we consider the case when $\operatorname{dim} L=4$.

Let $P_{n}(L)=\operatorname{ker} \delta^{n}$. Since $L$ is conilpotent, $P_{i-1}(L) \neq P_{i}(L)$ if $P_{i-1}(L) \neq L$.

If $\operatorname{dim} P_{1}(L)=4$, then $L$ is a 4 -dimensional Lie algebra with trivial $\delta$-structure.

If $\operatorname{dim} P_{1}(L)=1$, then $\operatorname{dim} P_{2}(L)=2$ with an element $x_{2} \in P_{2}(L) \backslash P_{1}(L)$ such that $\delta\left(x_{2}\right)=x_{1} \otimes x_{1}$. For any $x_{3} \in P_{3}(L) \backslash P_{2}(L)$, write $\delta\left(x_{3}\right)=a x_{1} \otimes x_{2}+b x_{2} \otimes$ $x_{1}+c x_{2} \otimes x_{2}$. The coassociativity on $x_{3}$ implies that $a=b$ and $c=0$. Without loss of generality, we assume that $\delta\left(x_{3}\right)=x_{1} \otimes x_{2}+x_{2} \otimes x_{1}$. Thus $x_{1}, x_{2}-\frac{1}{2} x_{1}^{2}$ and $x_{3}-x_{1} x_{2}+\frac{1}{3} x_{1}^{3}$ are linearly independent primitive elements in $U(L)$. Thus $\operatorname{dim} P(U(L))$ is at least 3. Hence $L$ is quasi-equivalent to an anti-cocommutative CLA by Theorem 2.7

Suppose $\operatorname{dim} P_{1}(L)=2$ with a basis $\left\{x_{1}, x_{2}\right\}$. If $\operatorname{dim} P(U(L)) \geq 3, L$ is quasiequivalent to an anti-commutative CLA by Theorem 2.7 . So we only need to consider the case when $\operatorname{dim} P(U(L))=2$. If $\operatorname{dim} P_{2}(L)=4$, choose two basis elements $y_{1}, y_{2}$ in $P_{2}(L) \backslash P_{1}(L)$. Then $\delta\left(y_{1}-f\left(x_{1}, x_{2}\right)\right)=a\left(x_{1} \otimes x_{2}-x_{2} \otimes x_{1}\right)$ and $\delta\left(y_{2}-g\left(x_{1}, x_{2}\right)\right)=b\left(x_{1} \otimes x_{2}-x_{2} \otimes x_{1}\right)$ for some polynomial $f\left(x_{1}, x_{2}\right)$ and $g\left(x_{1}, x_{2}\right)$. Since $P(U(L))=2$, both $a$ and $b$ are nonzero. Thus $a\left(y_{2}-g\right)-b\left(y_{1}-f\right)$ is an extra primitive element, a contradiction. Hence $\operatorname{dim} P_{2}(L)=3$ and $\operatorname{dim} P_{3}(L)=4$. Let $x_{3}$ be a basis element in $P_{2}(L)$ and $x_{4}$ be a basis element in $P_{3}(L)$. Write $\delta\left(x_{4}\right)=f \otimes x_{3}+x_{3} \otimes g+a x_{3} \otimes x_{3}$ with $f, g \in P_{1}(L)$ and $a \in k$. Coassociativity on $x_{4}$ implies that $a=0, f=g \neq 0$ and $\delta\left(x_{3}\right)=b f \otimes f$ for some $b \in k$. Thus $L$ is symmetric and hence quasi-equivalent to a Lie algebra by [WZZ3, Corollary 2.6]. 
If $\operatorname{dim} P_{1}(L)=3$, then $\operatorname{dim} P(U(L))$ is at least 3 and the assertion follows from Theorem 2.7

Before we continue we would like to make a remark about the lantern of connected Hopf algebras of GK-dimension 5, which could serve as a guideline for the classification of connected Hopf algebras of GK-dimension 5.

Remark 2.10. Let $H$ be a connected Hopf algebra of GK-dimension 5 .

(a) Let $p(H)$ denote the dimension of the space of all primitive elements. Then $p(H)$ is in the range $[2,5]$.

(b) If $p(H)=5$, then $\mathfrak{L}(H)$ is abelian (which is unique) and $H$ is isomorphic to the enveloping algebra over a Lie algebra.

(c) If $p(H)=4$, then $\mathfrak{L}(H)$ is generated by four elements in degree 1 (there are two such graded Lie algebras up to isomorphism) and $H$ is isomorphic to the enveloping algebra over an anti-cocommutative coassociative Lie algebra.

(d) If $p(H)=3$, then $\mathfrak{L}(H)$ is generated by three elements in degree 1 and there are two subcases.

(d1) If the degree two component of $\mathfrak{L}(H)$ has dimension 2 (there is only one such graded Lie algebra), then $H$ is possibly isomorphic to an enveloping algebra over an anti-cocommutative coassociative Lie algebra.

(d2) If the degree two component of $\mathfrak{L}(H)$ has dimension 1 (there is only one such graded Lie algebra), then $H$ is not isomorphic to the enveloping algebra over either an ordinary Lie algebra or a coassociative Lie algebra, nor over a primitively-thin Hopf algebra.

(e) If $p(H)=2$, then $\mathfrak{L}(H)$ is generated by two elements in degree 1 (there are two such graded Lie algebras), and $H$ is a primitively-thin Hopf algebra.

\section{Classification of anti-Cocommutative ClAs up to Dimension 4}

In this section we classify all anti-cocommutative CLAs of dimension up to 4 . The case of dimension 1 is trivial.

3.1. Dimension 2. We start with an easy observation.

Lemma 3.1. Let $L$ be an anti-cocommutative $C L A$ of dimension 2. Then $\delta=0$ and $L$ is an ordinary Lie algebra.

Proof. Let $I=\operatorname{ker} \delta$. By [WZZ3, Lemma 2.8(b)], $\delta(L) \subset I^{\otimes 2}$. If $I=0$, then $\delta=0$. If $\operatorname{dim} I=1$, pick a nonzero element $x \in I$. Then, for every $y \in L, \delta(y)=\lambda x \otimes x$ for some $\lambda \in k$. By the anti-cocommutativity of $\delta, \lambda=0$. Thus $\delta=0$. The remaining case is when $\operatorname{dim} I=2$, which implies that $\delta=0$.

It is well known that a 2-dimensional Lie algebra is either abelian or solvable with $[x, y]=y$ for a suitable basis $\{x, y\}$.

3.2. Dimension 3. The following lemma is similar to Lemma 3.1 The classification of 3-dimensional Lie algebras is well known, and is called the Bianchi classification when the base field is either the real numbers $\mathbb{R}$ or the complex numbers $\mathbb{C}$ (for instance, see [OV, p. 209, Theorem 1.1] if $k=\mathbb{C}$ ). We will not include the list here. Next we will consider those CLAs with nontrivial $\delta$. 
Let $a\left(\lambda_{1}, \lambda_{2}, \alpha\right)$ denote the CLA with a basis $\{x, y, z\}$ whose Lie algebra structure on $L=: B(b)$ is determined by

$$
\begin{aligned}
& {[x, y]=0,} \\
& {[z, x]=\lambda_{1} x+\alpha y,} \\
& {[z, y]=\lambda_{2} y}
\end{aligned}
$$

for some $\lambda_{1}, \lambda_{2}, \alpha \in k$, and whose coalgebra structure is determined by $\delta(x)=$ $\delta(y)=0$ and $\delta(z)=x \otimes y-y \otimes x$.

Lemma 3.2. Let $L$ be an anti-cocommutative $C L A$ of dimension 3 such that $\delta \neq 0$. Then $L$ is isomorphic to one of the following:

(a) $a(0,0,0)$.

(b) $a(1, \lambda, 0)$, and $a\left(1, \lambda^{\prime}, 0\right)$ is isomorphic to $a(1, \lambda, 0)$ if and only if $\lambda^{\prime}=\lambda$ or $\lambda^{-1}$.

(c) $a(0,0,1)$.

(d) $a(1,1,1)$.

(e) The CLA $b(\lambda)$ with a basis $\{x, y, z\}$, whose Lie algebra structure is determined by

$$
\begin{aligned}
& {[x, y]=y,} \\
& {[z, x]=-z+\lambda y,} \\
& {[z, y]=0,}
\end{aligned}
$$

where $\lambda$ is in $k$, and whose coalgebra structure is determined by $\delta(x)=$ $\delta(y)=0$ and $\delta(z)=x \otimes y-y \otimes x$, and $b\left(\lambda^{\prime}\right)$ is isomorphic to $b(\lambda)$ if and only if $\lambda^{\prime}=\lambda$.

All CLAs listed above are pairwise nonisomorphic except for the isomorphisms given in part (b).

Proof. Let $I=\operatorname{ker} \delta$. Repeating the first part of the proof of Lemma 3.1, one sees that $\operatorname{dim} I>1$. Since $\operatorname{dim} L=3$ and $\delta \neq 0$, we have $\operatorname{dim} I=2$. Pick any basis $\{x, y\}$ of $I$. The only skew-symmetric elements in $I^{\otimes 2}$ are $\lambda(x \otimes y-y \otimes x)$ for some $\lambda \in k^{\times}:=k \backslash\{0\}$. Let $z \in L \backslash I$ such that $\delta(z)=x \otimes y-y \otimes x$.

Since $I$ is a Lie subalgebra of $L$ (see Lemma 2.5(e)), we have the following two cases to consider.

Case 1: $I$ is abelian. Write

$$
\begin{aligned}
& {[z, x]=a_{1} x+a_{2} y+a_{3} z} \\
& {[z, y]=b_{1} x+b_{2} y+b_{3} z}
\end{aligned}
$$

Applying (E2.1.1) to $(a, b)=(z, x)$, we have

$$
a_{3}(x \otimes y-y \otimes x)=\delta([z, x])=[\delta(z), x \otimes 1+1 \otimes x]=0 .
$$

Hence $a_{3}=0$. By symmetry, $b_{3}=0$.

Using a linear transformation $f: x \rightarrow c_{11} x+c_{12} y, y \rightarrow c_{21} x+c_{22} y$ with $\operatorname{det}\left(\begin{array}{ll}c_{11} & c_{12} \\ c_{21} & c_{22}\end{array}\right)=1$, the matrix $\left(\begin{array}{ll}a_{1} & a_{2} \\ b_{1} & b_{2}\end{array}\right)$ becomes one of the Jordan forms

$$
\left(\begin{array}{ll}
a & 0 \\
0 & b
\end{array}\right) \text { or }\left(\begin{array}{ll}
a & 1 \\
0 & a
\end{array}\right) \text {. }
$$


In the first Jordon case, if $a=b=0$, this is case (a). If $a \neq 0$ (or by symmetry if $b \neq 0$ ) we can assume that $a=1$ by a change of basis $\{x, y, z\} \rightarrow$ $\left\{\sqrt{a^{-1}} x, \sqrt{a^{-1}} y, a^{-1} z\right\}$. So this is case (b). In the second Jordon case, if $a=0$, this is case (c). If $a \neq 0$, by a change of basis, we can assume that $a=1$, which is case $(\mathrm{d})$.

Case 2: $I$ is not abelian. So we may assume that $[x, y]=y$ where $\{x, y\}$ is a basis of $I$. Let $z \in L \backslash I$ such that $\delta(z)=x \otimes y-y \otimes x$. Write

$$
\begin{aligned}
& {[z, x]=a_{1} x+a_{2} y+a_{3} z,} \\
& {[z, y]=b_{1} x+b_{2} y+b_{3} z .}
\end{aligned}
$$

Applying (E2.1.1) to $(a, b)=(z, x)$, we have

$$
a_{3}(x \otimes y-y \otimes x)=\delta([z, x])=[\delta(z), x \otimes 1+1 \otimes x]=-(x \otimes y-y \otimes x) .
$$

Hence $a_{3}=-1$. A similar argument shows that $b_{3}=0$. By the Jacobi identity, we have

$$
\begin{aligned}
b_{1} x+b_{2} y & =[z, y]=[z,[x, y]]=[[z, x], y]+[x,[z, y]] \\
& =\left[a_{1} x+a_{2} y-z, y\right]+\left[x, b_{1} x+b_{2} y\right] \\
& =a_{1} y-\left(b_{1} x+b_{2} y\right)+b_{2} y .
\end{aligned}
$$

Then $b_{1}=0$ and $a_{1}=b_{2}$. Thus we have

$$
\begin{aligned}
& {[z, x]=a x+a_{2} y-z,} \\
& {[z, y]=a y .}
\end{aligned}
$$

After replacing $z$ by $z-a x$, we have $a=0$. This is case (e).

By the above argument, the CLAs listed are pairwise nonisomorphic except for the isomorphism given in part (b).

The enveloping algebra of case (e) has an interesting property that $S^{2}$ is not the identity; see [WZZ3, Example 4.2].

3.3. Dimension 4. This is the main subsection of Section 3. We will classify all 4-dimensional anti-cocommutative CLAs. If $\delta=0, L$ is an ordinary Lie algebra of dimension 4 and the classification is known OV, p. 209, Theorem 1.1] in which the base field $k$ is $\mathbb{C}$. So we assume that $\delta \neq 0$. Throughout this subsection we assume that $L$ is a 4 -dimensional anti-commutative CLA such that $\delta \neq 0$.

Lemma 3.3. Let $I=\operatorname{ker} \delta$. Then $I$ is a 3-dimensional Lie subalgebra of $L$.

Proof. An easy calculation shows that $I$ is a Lie subalgebra of $L$ by using (E2.1.1). It remains to show that $\operatorname{dim} I>2$. Let $H=U(L)$. By Lemma $2.5(\mathrm{~g}), p_{2}^{\prime}(H)=$ $\operatorname{dim} L-\operatorname{dim} I \leq\left(\begin{array}{c}\operatorname{dim} I \\ 2\end{array}\right)$. If $\operatorname{dim} I \leq 2$, then $p_{2}^{\prime}(H) \leq 1$ and $\operatorname{dim} L \leq 3$, a contradiction. Therefore $\operatorname{dim} I>2$.

Lemma 3.4. There are elements $x_{1}, x_{2}$ in I such that $\delta(L)=k\left(x_{1} \otimes x_{2}-x_{2} \otimes x_{1}\right)$.

Proof. Let $\left\{x_{1}^{\prime}, x_{2}^{\prime}, x_{3}^{\prime}\right\}$ be a basis of $I$. Since $\operatorname{dim} I=3, \operatorname{dim} \delta(L)=1$. Pick $z \in L \backslash I$. Then we must have

$$
\delta(z)=\sum_{i, j} a_{i j}\left(x_{i}^{\prime} \otimes x_{j}^{\prime}-x_{j}^{\prime} \otimes x_{i}^{\prime}\right),
$$

where $A=\left(a_{i j}\right)$ is a nonzero $3 \times 3$ anti-symmetric matrix. Obviously, $\delta(L)$ is spanned by $\delta(z)$. Now $A$ has eigenvalues $0, \lambda,-\lambda$ for some $\lambda \neq 0$. By replacing $z$ 
with $z / \sqrt[3]{\lambda}$, we can assume that $A$ has eigenvalues $0,1,-1$. By linear algebra, there exists an invertible matrix $P$ such that

$$
P A P^{T}=\left(\begin{array}{ccc}
0 & 1 & 0 \\
-1 & 0 & 0 \\
0 & 0 & 0
\end{array}\right) .
$$

By setting $\left(x_{1}, x_{2}, x_{3}\right)^{T}=P^{-1}\left(x_{1}^{\prime}, x_{2}^{\prime}, x_{3}^{\prime}\right)^{T}$, we get

$$
\delta(z)=x_{1} \otimes x_{2}-x_{2} \otimes x_{1} .
$$

This completes the proof.

Here is the main result of this section. The proof is by computation, and some details are easy to check.

Theorem 3.5. Let $L$ be an anti-cocommutative CLA of dimension 4 such that $\delta \neq 0$. Then there is a basis $\left\{x_{1}, x_{2}, x_{3}, z\right\}$ such that the coalgebra structure is given by $\delta\left(x_{i}\right)=0$ and $\delta(z)=x_{1} \otimes x_{2}-x_{2} \otimes x_{1}$. The Lie algebra structure of $L$ is given by one of the following:

(a)

$$
\begin{aligned}
{\left[x_{2}, x_{1}\right] } & =x_{2}, \\
{\left[x_{3}, x_{1}\right] } & =\left[x_{3}, x_{2}\right]=0, \\
{\left[z, x_{1}\right] } & =z+a x_{1}+c x_{2}, \\
{\left[z, x_{2}\right] } & =a x_{2}, \\
{\left[z, x_{3}\right] } & =b x_{2},
\end{aligned}
$$

where $(a, b)=(1,1),(1,0),(0,1)$ or $(0,0)$ and $c \in k$.

(b) $L=B\left(\left(a_{i j}\right)\right)$ is determined by

$$
\begin{aligned}
{\left[x_{2}, x_{1}\right] } & =\left[x_{3}, x_{1}\right]=\left[x_{3}, x_{2}\right]=0, \\
{\left[z, x_{1}\right] } & =a_{11} x_{1}+a_{12} x_{2}+a_{13} x_{3}, \\
{\left[z, x_{2}\right] } & =a_{21} x_{1}+a_{22} x_{2}+a_{23} x_{3}, \\
{\left[z, x_{3}\right] } & =a_{31} x_{1}+a_{32} x_{2}+a_{33} x_{3},
\end{aligned}
$$

where $\left(a_{i j}\right)$ is a $3 \times 3$ matrix over $k$. The CLA $B\left(\left(a_{i j}\right)\right)$ is isomorphic to $B\left(\left(b_{i j}\right)\right)$ if and only if the matrix $\left(a_{i j}\right)$ is similar to $\lambda\left(b_{i j}\right)$ for some $\lambda \in k^{\times}$. (c)

$$
\begin{aligned}
{\left[x_{2}, x_{1}\right] } & =0, \\
{\left[x_{3}, x_{1}\right] } & =x_{2}, \\
{\left[x_{3}, x_{2}\right] } & =0, \\
{\left[z, x_{1}\right] } & =a x_{1}+b x_{3}, \\
{\left[z, x_{2}\right] } & =x_{2}, \\
{\left[z, x_{3}\right] } & =c x_{1}+(1-a) x_{3},
\end{aligned}
$$

where $a, b, c \in k$. 
(d)

$$
\begin{aligned}
{\left[x_{2}, x_{1}\right] } & =0, \\
{\left[x_{3}, x_{1}\right] } & =x_{2}, \\
{\left[x_{3}, x_{2}\right] } & =0, \\
{\left[z, x_{1}\right] } & =a x_{1}+b x_{3}, \\
{\left[z, x_{2}\right] } & =0, \\
{\left[z, x_{3}\right] } & =c x_{1}-a x_{3},
\end{aligned}
$$

where $a, b, c \in k$.

(e)

$$
\begin{aligned}
{\left[x_{2}, x_{1}\right] } & =\left[x_{3}, x_{2}\right]=0, \\
{\left[x_{3}, x_{1}\right] } & =x_{1}, \\
{\left[z, x_{1}\right] } & =a x_{1}, \\
{\left[z, x_{2}\right] } & =b x_{1}, \\
{\left[z, x_{3}\right] } & =-z+c x_{1}+a x_{3},
\end{aligned}
$$

(f)

where $(a, b)=(1,1),(1,0),(0,1)$ or $(0,0)$ and $c \in k$.

$$
\begin{aligned}
{\left[x_{2}, x_{1}\right] } & =0, \\
{\left[x_{3}, x_{1}\right] } & =x_{1}+x_{2}, \\
{\left[x_{3}, x_{2}\right] } & =x_{2}, \\
{\left[z, x_{1}\right] } & =\left[z, x_{2}\right]=0, \\
{\left[z, x_{3}\right] } & =-2 z .
\end{aligned}
$$

(g)

$$
\begin{aligned}
{\left[x_{2}, x_{1}\right] } & =0, \\
{\left[x_{3}, x_{1}\right] } & =x_{1}, \\
{\left[x_{3}, x_{2}\right] } & =-x_{2}, \\
{\left[z, x_{1}\right] } & =a x_{1}+c x_{2}, \\
{\left[z, x_{2}\right] } & =b x_{1}, \\
{\left[z, x_{3}\right] } & =0,
\end{aligned}
$$

where $(a, b)=(1,1),(1,0),(0,1)$ or $(0,0)$ and $c \in k$.

(h) $L=H(\lambda, a)$ is determined by

$$
\begin{aligned}
{\left[x_{2}, x_{1}\right] } & =0, \\
{\left[x_{3}, x_{1}\right] } & =x_{1}, \\
{\left[x_{3}, x_{2}\right] } & =\lambda x_{2}, \\
{\left[z, x_{1}\right] } & =a x_{2}, \\
{\left[z, x_{2}\right] } & =a x_{1}, \\
{\left[z, x_{3}\right] } & =(-1-\lambda) z,
\end{aligned}
$$

where $\lambda \in k \backslash\{0,-1\}$ and $a \in\{0,1\}$. The CLA $H(\lambda, a)$ is isomorphic to $H\left(\lambda^{\prime}, a^{\prime}\right)$ if and only if $a^{\prime}=a$ and $\lambda^{\prime}=\lambda$ or $\lambda^{\prime}=\lambda^{-1}$.

The CLAs listed above are pairwise nonisomorphic except for the isomorphisms given in parts $(\mathrm{b}, \mathrm{h})$. 
Proof. By the previous two lemmas, for any 4-dimensional anti-cocommutative CLA $L$ with nonzero $\delta$, we can choose a basis $\left\{x_{1}, x_{2}, x_{3}, z\right\}$ such that $I=\operatorname{ker} \delta$ is spanned by $\left\{x_{1}, x_{2}, x_{3}\right\}$ and $\delta(z)=x_{1} \otimes x_{2}-x_{1} \otimes x_{2}$.

Write

$$
\begin{aligned}
{\left[x_{2}, x_{1}\right] } & =a_{1} x_{1}+b_{1} x_{2}+c_{1} x_{3}, \\
{\left[x_{3}, x_{1}\right] } & =a_{2} x_{1}+b_{2} x_{2}+c_{2} x_{3}, \\
{\left[x_{3}, x_{2}\right] } & =a_{3} x_{1}+b_{3} x_{2}+c_{3} x_{3}, \\
{\left[z, x_{1}\right] } & =e_{1} z+\phi_{1}=e_{1} z+f_{1} x_{1}+g_{1} x_{2}+h_{1} x_{3}, \\
{\left[z, x_{2}\right] } & =e_{2} z+\phi_{2}=e_{2} z+f_{2} x_{1}+g_{2} x_{2}+h_{2} x_{3}, \\
{\left[z, x_{3}\right] } & =e_{3} z+\phi_{3}=e_{3} z+f_{3} x_{1}+g_{3} x_{2}+h_{3} x_{3} .
\end{aligned}
$$

Applying (E2.1.1) to $\left(z, x_{i}\right)$ for $i=1,2,3$, one obtains that $c_{1}=c_{2}=c_{3}=0$ and that $e_{1}=b_{1}, e_{2}=-a_{1}, e_{3}=-a_{2}-b_{3}$. Thus (E2.1.1) holds if and only if the Lie bracket satisfies

$$
\begin{aligned}
{\left[x_{2}, x_{1}\right] } & =a_{1} x_{1}+b_{1} x_{2}, \\
{\left[x_{3}, x_{1}\right] } & =a_{2} x_{1}+b_{2} x_{2}, \\
{\left[x_{3}, x_{2}\right] } & =a_{3} x_{1}+b_{3} x_{2}, \\
{\left[z, x_{1}\right] } & =b_{1} z+f_{1} x_{1}+g_{1} x_{2}+h_{1} x_{3}, \\
{\left[z, x_{2}\right] } & =-a_{1} z+f_{2} x_{1}+g_{2} x_{2}+h_{2} x_{3}, \\
{\left[z, x_{3}\right] } & =\left(-a_{2}-b_{3}\right) z+f_{3} x_{1}+g_{3} x_{2}+h_{3} x_{3} .
\end{aligned}
$$

In particular, $J:=k x_{1}+k x_{2}$ is a Lie subalgebra. So $J$ is either abelian or solvable.

If $J$ is solvable, we may assume that $\left[x_{2}, x_{1}\right]=x_{2}$. Since $I$ is a Lie subalgebra,

$$
\begin{aligned}
a_{3} x_{1}+b_{3} x_{2} & =\left[x_{3}, x_{2}\right]=\left[x_{3},\left[x_{2}, x_{1}\right]\right] \\
& =\left[\left[x_{3}, x_{2}\right], x_{1}\right]+\left[x_{2},\left[x_{3}, x_{1}\right]\right] \\
& =\left[a_{3} x_{1}+b_{3} x_{2}, x_{1}\right]+\left[x_{2}, a_{2} x_{1}+b_{2} x_{2}\right] \\
& =b_{3} x_{2}+a_{2} x_{2} .
\end{aligned}
$$

This implies that $a_{2}=a_{3}=0$.

If $b_{2} \neq 0$ or $b_{3} \neq 0$, after a base change, we have $b_{2}=b_{3}=0$. The Jacobi identity for other elements implies that

$$
\begin{aligned}
{\left[x_{2}, x_{1}\right] } & =x_{2}, \\
{\left[x_{3}, x_{1}\right] } & =0, \\
{\left[x_{3}, x_{2}\right] } & =0, \\
{\left[z, x_{1}\right] } & =z+f_{1} x_{1}+g_{1} x_{2}+h_{1} x_{3}, \\
{\left[z, x_{2}\right] } & =f_{1} x_{2}, \\
{\left[z, x_{3}\right] } & =g_{3} x_{2},
\end{aligned}
$$

where $f_{i}, g_{i}, h_{i} \in k$. There are a few cases. First of all, we can make $h_{1}=0$ by replacing $z$ with $z+h_{1} x_{3}$. If $f_{1} \neq 0$, we can assume $f_{1}=1$ by replacing $z, x_{2}$ with $\frac{1}{f_{1}} z, \frac{1}{f_{1}} x_{2}$, respectively. If $g_{3} \neq 0$, we can also normalize it to be 1 by replacing $x_{3}$ with $\frac{1}{g_{3}} x_{3}$. All nonisomorphic Lie algebras are now listed in part (a).

If $J$ is abelian, $\left[x_{2}, x_{1}\right]=0$. If $I$ is abelian, then $\left[z, x_{i}\right]=\phi_{i}$ defines a Lie algebra and for any $\phi_{i} \in I, i=1,2,3$. This is part (b). Further classification can be made 
by taking the Jordan form of the coefficient matrix of $\left\{\phi_{1}, \phi_{2}, \phi_{3}\right\}$. This is a linear algebra classification and, to save space, we will not list all the possibilities.

For the rest of the proof we assume that $J$ is abelian and $I$ is not abelian. Up to a change of basis $\left\{x_{1}, x_{2}\right\}$, we may assume that

$$
\begin{aligned}
& {\left[x_{3}, x_{1}\right]=a x_{1}+b x_{2},} \\
& {\left[x_{3}, x_{2}\right]=c x_{2},}
\end{aligned}
$$

where

$$
\left(\begin{array}{ll}
a & b \\
0 & c
\end{array}\right)=\left(\begin{array}{ll}
0 & 1 \\
0 & 0
\end{array}\right) \text { or }\left(\begin{array}{ll}
1 & 0 \\
0 & 0
\end{array}\right) \text { or }\left(\begin{array}{ll}
1 & 1 \\
0 & 1
\end{array}\right) \text { or }\left(\begin{array}{ll}
1 & 0 \\
0 & \lambda
\end{array}\right)
$$

where $\lambda \in k^{\times}$.

Recall that

$$
\begin{aligned}
& {\left[z, x_{1}\right]=\phi_{1}=f_{1} x_{1}+g_{1} x_{2}+h_{1} x_{3},} \\
& {\left[z, x_{2}\right]=\phi_{2}=f_{2} x_{1}+g_{2} x_{2}+h_{2} x_{3},} \\
& {\left[z, x_{3}\right]=(-a-c) z+\phi_{3}=(-a-c) z+f_{3} x_{1}+g_{3} x_{2}+h_{3} x_{3} .}
\end{aligned}
$$

The Jacobi identity implies that

$$
\begin{aligned}
0 & =\left[\phi_{2}, x_{1}\right]+\left[x_{2}, \phi_{1}\right], \\
(2 a+c) \phi_{1}+b \phi_{2} & =\left[\phi_{3}, x_{1}\right]+\left[x_{3}, \phi_{1}\right], \\
(a+2 c) \phi_{2} & =\left[\phi_{3}, x_{2}\right]+\left[x_{3}, \phi_{2}\right],
\end{aligned}
$$

which completely determine the Lie algebra $L$.

If $\left(\begin{array}{ll}a & b \\ 0 & c\end{array}\right)=\left(\begin{array}{ll}0 & 1 \\ 0 & 0\end{array}\right)$, then we have

$$
\begin{aligned}
{\left[x_{2}, x_{1}\right] } & =0, \\
{\left[x_{3}, x_{1}\right] } & =x_{2}, \\
{\left[x_{3}, x_{2}\right] } & =0, \\
{\left[z, x_{1}\right] } & =f_{1} x_{1}+g_{1} x_{2}+h_{1} x_{3}, \\
{\left[z, x_{2}\right] } & =\left(h_{3}+f_{1}\right) x_{2}, \\
{\left[z, x_{3}\right] } & =f_{3} x_{1}+g_{3} x_{2}+h_{3} x_{3} .
\end{aligned}
$$

First, we can make $g_{1}=g_{3}=0$ by replacing $z$ with $z+g_{3} x_{1}-g_{1} x_{3}$. If $h_{3}+f_{1} \neq 0$, we can make $h_{3}+f_{1}=1$ by replacing $x_{1}, x_{2}, z$ with $\frac{1}{\sqrt{h_{3}+f_{1}}} x_{1}, \frac{1}{\sqrt{h_{3}+f_{1}}} x_{2}, \frac{1}{h_{3}+f_{1}} z$. After recycling the letters $a, b, c$, we obtain part (c). If $h_{3}+f_{1}=0$, this is part (d) after recycling the letters $a, b, c$.

For the rest we use similar computations, so some details are omitted.

If $\left(\begin{array}{ll}a & b \\ 0 & c\end{array}\right)=\left(\begin{array}{ll}1 & 0 \\ 0 & 0\end{array}\right)$, then we have

$$
\begin{aligned}
{\left[x_{2}, x_{1}\right] } & =0, \\
{\left[x_{3}, x_{1}\right] } & =x_{1}, \\
{\left[x_{3}, x_{2}\right] } & =0, \\
{\left[z, x_{1}\right] } & =f_{1} x_{1}, \\
{\left[z, x_{2}\right] } & =f_{2} x_{1}, \\
{\left[z, x_{3}\right] } & =-z+f_{3} x_{1}+g_{3} x_{2}+f_{1} x_{3} .
\end{aligned}
$$


First, $g_{3}$ can be made 0 by replacing $z$ with $z-g_{3} x_{2}$. If $f_{1} \neq 0$, we can assume $f_{1}=1$ by replacing $z, x_{1}$ with $\frac{1}{f_{1}} z, \frac{1}{f_{1}} x_{1}$, respectively. If $f_{2} \neq 0$, we can also normalize it to be 1 by replacing $x_{1}, x_{2}$ with $\sqrt{f_{2}} x_{1}, \frac{1}{\sqrt{f_{2}}} x_{2}$, respectively. All cases are listed in part (e).

$$
\text { If } \begin{aligned}
&\left(\begin{array}{ll}
a & b \\
0 & c
\end{array}\right)=\left(\begin{array}{ll}
1 & 1 \\
0 & 1
\end{array}\right), \text { then we have } \\
& {\left[x_{2}, x_{1}\right] }=0 \\
& {\left[x_{3}, x_{1}\right] }=x_{1}+x_{2}, \\
& {\left[x_{3}, x_{2}\right] }=x_{2}, \\
& {\left[z, x_{1}\right] }=f_{1} x_{1}+f_{1} x_{2}, \\
& {\left[z, x_{2}\right] }=f_{1} x_{2}, \\
& {\left[z, x_{3}\right] }=-2 z+f_{3} x_{1}+g_{3} x_{2}+2 f_{1} x_{3} .
\end{aligned}
$$

First, we can make $f_{3}=g_{3}=0$ by replacing $z$ with $z-f_{3} x_{1}-\left(f_{3}+g_{3}\right) x_{2}$. Then we can assume that $f_{1}=0$ by replacing $z$ with $z-f_{1} x_{3}$. This is part (f).

Finally, if $\left(\begin{array}{ll}a & b \\ 0 & c\end{array}\right)=\left(\begin{array}{ll}1 & 0 \\ 0 & \lambda\end{array}\right)$ where $\lambda \neq 0$, then we have

$$
\begin{aligned}
{\left[x_{2}, x_{1}\right] } & =0, \\
{\left[x_{3}, x_{1}\right] } & =x_{1}, \\
{\left[x_{3}, x_{2}\right] } & =\lambda x_{2}, \\
{\left[z, x_{1}\right] } & =f_{1} x_{1}+g_{1} x_{2}, \\
{\left[z, x_{2}\right] } & =f_{2} x_{1}+g_{2} x_{2}, \\
{\left[z, x_{3}\right] } & =(-1-\lambda) z+f_{3} x_{1}+g_{3} x_{2}+(1+\lambda) f_{1} x_{3},
\end{aligned}
$$

such that $(1+\lambda) g_{1}=(1+\lambda) f_{2}=0$ and $\lambda(1+\lambda) f_{1}=(1+\lambda) g_{2}$.

Suppose $\lambda=-1$. Then we can make $g_{2}=f_{3}=g_{3}=0$ by replacing $z$ with $z+f_{3} x_{1}-g_{3} x_{2}+g_{2} x_{3}$. Then, if $f_{1} \neq 0$, we can make $f_{1}=1$ by replacing $x_{2}, z$ with $\frac{1}{f_{1}} x_{2}, \frac{1}{f_{1}} z$. If $f_{2} \neq 0$, we can make $f_{2}=1$ by replacing $x_{1}, x_{2}$ with $\sqrt{f_{2}} x_{1}, \frac{1}{\sqrt{f_{2}}} x_{2}$ (notice that $f_{1}$ will not change). This is part (g).

Suppose $\lambda \neq-1$. We can also assume that $f_{3}=g_{3}=0$ by replacing $z$ with $z-\frac{f_{3}}{\lambda} x_{1}-g_{3} x_{2}$. Now the last three relations become

$$
\begin{aligned}
& {\left[z, x_{1}\right]=f_{1} x_{1}+g_{1} x_{2},} \\
& {\left[z, x_{2}\right]=g_{1} x_{1}+\lambda f_{1} x_{2},} \\
& {\left[z, x_{3}\right]=(-1-\lambda) z+(1+\lambda) f_{1} x_{3} .}
\end{aligned}
$$

Then by replacing $z$ with $z-f_{1} x_{3}$, we can assume that $f_{1}=0$. This is part (h). We finish the proof.

\section{Classification of primitively-thin algebras OF DIMENSION AT MOST 4}

Recall that a connected Hopf algebra $H$ is primitively-thin if $p(H)=2$.

If $\operatorname{GKdim} H=2$, then $H=U(\mathfrak{g})$ for a Lie algebra of dimension 2, which follows from Lemmas 1.3(e) and 2.6(c). 
4.1. Primitively-thin Hopf algebras of GK-dimension 3. We recall the definition of two classes of Hopf algebras from [Zh2, which will be used later in the classification.

Example 4.1. Let $A$ be the algebra generated by elements $X, Y, Z$ satisfying the following relations:

$$
\begin{aligned}
& {[X, Y]=0,} \\
& {[Z, X]=\lambda_{1} X+\alpha Y,} \\
& {[Z, Y]=\lambda_{2} Y,}
\end{aligned}
$$

where $\alpha=0$ if $\lambda_{1} \neq \lambda_{2}$ and $\alpha=0$ or 1 if $\lambda_{1}=\lambda_{2}$. Then $A$ becomes a Hopf algebra via

$$
\begin{aligned}
& \epsilon(X)=0, \quad \Delta(X)=1 \otimes X+X \otimes 1, \\
& \epsilon(Y)=0, \quad \Delta(Y)=1 \otimes Y+Y \otimes 1, \\
& \epsilon(Z)=0, \quad \Delta(Z)=1 \otimes Z+X \otimes Y-Y \otimes X+Z \otimes 1 .
\end{aligned}
$$

We denote this Hopf algebra by $A\left(\lambda_{1}, \lambda_{2}, \alpha\right)$. It is easy to see that $A\left(\lambda_{1}, \lambda_{2}, \alpha\right)$ is the enveloping algebra $\left.U\left(a\left(\lambda_{1}, \lambda_{2}, \alpha\right)\right)\right)$ where $a\left(\lambda_{1}, \lambda_{2}, \alpha\right)$ is the CLA defined before Lemma 3.2 .

Example 4.2. Let $B$ be the algebra generated by elements $X, Y, Z$ satisfying the following relations:

$$
\begin{aligned}
& {[X, Y]=Y,} \\
& {[Z, X]=-Z+\lambda Y,} \\
& {[Z, Y]=0,}
\end{aligned}
$$

where $\lambda \in k$. Then $B$ becomes a Hopf algebra via the coalgebra structure given as in (E4.1.1). We denote this Hopf algebra by $B(\lambda)$. This algebra is the enveloping algebra $U(b(\lambda))$ where $b(\lambda)$ is the CLA defined in Lemma 3.2(e).

The following proposition is from [Zh2, Theorem 7.8]. It also follows now from Lemma 3.2 and Theorem 2.7.

Proposition 4.3. Let $H$ be a connected Hopf algebra. Then $H$ is primitively-thin of GK-dimension 3 if and only if $H$ is isomorphic to one of the following:

(a) the Hopf algebras $A(0,0,0), A(0,0,1), A(1,1,1)$ or $A(1, \lambda, 0)$ from Example 4.1 for some $\lambda \in k$;

(b) the Hopf algebras $B(\lambda)$ from Example 4.2 for some $\lambda \in k$.

4.2. Examples of connected Hopf algebras of GK-dimension 4. We introduce four classes of connected Hopf algebras of GK-dimension 4. Later we will show that these classes give a complete description of primitively-thin Hopf algebras of GK-dimension 4.

Example 4.4. Let $D$ be the algebra generated by $X, Y, Z, W$ satisfying the following relations:

$$
\begin{aligned}
{[Y, X] } & =[Z, X]=[Z, Y]=0, \\
{[W, X] } & =a_{11} X+a_{12} Y, \\
{[W, Y] } & =a_{21} X+a_{22} Y, \\
{[W, Z] } & =\left(a_{11}+a_{22}\right) Z+\xi_{1} X+\xi_{2} Y,
\end{aligned}
$$


where $a_{i j}, \xi_{i} \in k$. Then $D$ becomes a bialgebra via

$$
\begin{aligned}
& \epsilon(X)=0, \quad \Delta(X)=1 \otimes X+X \otimes 1, \\
& \epsilon(Y)=0, \quad \Delta(Y)=1 \otimes Y+Y \otimes 1, \\
& \epsilon(Z)=0, \quad \Delta(Z)=1 \otimes Z+X \otimes Y-Y \otimes X+Z \otimes 1, \\
& \epsilon(W)=0 \text {, } \\
& \Delta(W)=1 \otimes W+W \otimes 1 \\
& +\theta_{1}(Z \otimes X-X \otimes Z+X \otimes X Y+X Y \otimes X) \\
& +\theta_{2}(Y \otimes Z-Z \otimes Y+X Y \otimes Y+Y \otimes X Y),
\end{aligned}
$$

where $\theta_{i} \in k$ and at least one of them is nonzero. This bialgebra is also denoted by $D\left(\left\{\theta_{i}\right\},\left\{a_{i j}\right\},\left\{\xi_{i}\right\}\right)$ if we want to indicate the parameters. It is easy to see that the coalgebra structure is connected. Hence the bialgebra $D$ is automatically a Hopf algebra. Note that $P(D)=k X+k Y$ and $P_{2}(D)=k X+k Y+k Z$. Let $f$ be a Hopf algebra isomorphism between two such Hopf algebras (or a Hopf algebra automorphism). Then $f$ preserves the subspaces $k X+k Y$ and $k X+k Y+k Z$, and it is now not hard to see that $f$ sends

$$
\begin{aligned}
& X \longrightarrow c_{11} X+c_{12} Y, \\
& Y \longrightarrow c_{21} X+c_{22} Y, \\
& Z \longrightarrow c_{31} X+c_{32} Y+c_{33} Z, \\
& W \longrightarrow c_{44} W+w(X, Y, Z),
\end{aligned}
$$

where $c_{i j} \in k$ and $w(X, Y, Z)$ is a certain polynomial of $X, Y, Z$. (Note that $f$ being a Hopf algebra isomorphism implies that $c_{i j}$ and $w$ satisfy some conditions for which we will not give details here.)

Using an isomorphism $f$, or equivalently, choosing a new basis $\{X, Y, Z, W\}$ properly, one can first normalize the matrix $\left(a_{i j}\right)_{2 \times 2}$ so that it becomes one of the following five:

$$
\left(\begin{array}{ll}
0 & 0 \\
0 & 0
\end{array}\right),\left(\begin{array}{ll}
0 & 1 \\
0 & 0
\end{array}\right),\left(\begin{array}{ll}
1 & 0 \\
0 & 0
\end{array}\right),\left(\begin{array}{ll}
1 & 1 \\
0 & 1
\end{array}\right),\left(\begin{array}{ll}
1 & 0 \\
0 & \lambda
\end{array}\right),
$$

where $\lambda \neq 0$ and in the last class $\left(\begin{array}{ll}1 & 0 \\ 0 & \lambda\end{array}\right)$ is equivalent to $\left(\begin{array}{cc}1 & 0 \\ 0 & \lambda^{-1}\end{array}\right)$. The Hopf algebras are pairwise nonisomorphic if these are in different classes. Within any class, two Hopf algebras $D\left(\left\{\theta_{i}\right\},\left\{a_{i j}\right\},\left\{\xi_{i}\right\}\right)$ can be isomorphic for different parameters $\left\{\theta_{i}\right\}$ and $\left\{\xi_{i}\right\}$, which is determined by the base changes that fix the matrix given in (E4.4.2) (or change $\left(\begin{array}{ll}1 & 0 \\ 0 & \lambda\end{array}\right)$ to $\left(\begin{array}{cc}1 & 0 \\ 0 & \lambda^{-1}\end{array}\right)$ if in the last class). For example, by replacing $\{X, Y, Z, W\}$ by $\left\{a X, a Y, a^{2} Z, W\right\}$, the parameters $\left\{\theta_{1}, \theta_{2}\right\}$ become $\left\{a^{-3} \theta_{1}, a^{-3} \theta_{2}\right\}$. This means that we may assume that $\left\{\theta_{1}, \theta_{2}\right\} \in \mathbb{P}^{1}$. Dependent on the form of the matrix $\left(a_{i j}\right)$ listed in (E4.4.2), one can further decide the parameters $\left\{\xi_{1}, \xi_{2}\right\}$ such that the Hopf algebras $D\left(\left\{\theta_{i}\right\},\left\{a_{i j}\right\},\left\{\xi_{i}\right\}\right)$ are nonisomorphic. In summary, the isomorphism classes of $D\left(\left\{\theta_{i}\right\},\left\{a_{i j}\right\},\left\{\xi_{i}\right\}\right)$ can be completely determined by easy linear algebra.

There is another way of classifying all isomorphism classes of $D\left(\left\{\theta_{i}\right\},\left\{a_{i j}\right\},\left\{\xi_{i}\right\}\right)$. First, by choosing the basis $\{X, Y, Z, W\}$ properly, one can assume that $\theta_{1}=0$ and 
$\theta_{2}=1$. So we can fix $\left\{\theta_{1}, \theta_{2}\right\}=\{0,1\}$. In particular,

$$
\Delta(W)=1 \otimes W+W \otimes 1+Y \otimes Z-Z \otimes Y+X Y \otimes Y+Y \otimes X Y .
$$

Under this restriction, one can further classify the parameters $\left\{\left(a_{i j}\right),\left\{\xi_{i}\right\}\right\}$. Unfortunately, then we cannot assume that the matrix $\left(a_{i j}\right)$ is of the form given in (E4.4.2).

From the algebraic relations, $D\left(\left\{\theta_{i}\right\},\left\{a_{i j}\right\},\left\{\xi_{i}\right\}\right)$ is isomorphic to a universal enveloping algebra of a Lie algebra.

Example 4.5. Let $E$ be the algebra generated by $X, Y, Z, W$ satisfying the following relations:

$$
\begin{aligned}
{[Y, X] } & =[Z, Y]=0 \\
{[Z, X] } & =X \\
{[W, X] } & =a X \\
{[W, Y] } & =b X \\
{[W, Z] } & =a Z-W+\xi X+\xi^{\prime} Y,
\end{aligned}
$$

where $\xi, \xi^{\prime} \in k$. Then $E$ becomes a bialgebra (and then a Hopf algebra) via

$$
\begin{aligned}
& \epsilon(X)=0, \Delta(X)=1 \otimes X+X \otimes 1, \\
& \epsilon(Y)=0, \Delta(Y)=1 \otimes Y+Y \otimes 1, \\
& \epsilon(Z)=0, \Delta(Z)=1 \otimes Z+X \otimes Y-Y \otimes X+Z \otimes 1, \\
& \epsilon(W)=0, \\
& \Delta(W)=1 \otimes W+W \otimes 1 \\
& \quad+Z \otimes X-X \otimes Z+X \otimes X Y+X Y \otimes X .
\end{aligned}
$$

Up to a base change (by setting $W_{\text {new }}=W-\xi^{\prime} Y$ ), we may assume that $\xi^{\prime}=0$. We denote this Hopf algebra by $E(a, b, \xi)$ where $a, b, \xi \in k$. Using a base change

$$
\begin{aligned}
X & \rightarrow c X, \\
Y & \rightarrow c^{-1} Y, \\
Z & \rightarrow Z, \\
W & \rightarrow c W,
\end{aligned}
$$

for some $c \in k$, we can rescale $(a, b, \xi)$. The complete set of nonisomorphic classes of $E(a, b, \xi)$ corresponds to the following cases:

$$
(a, b, \xi)= \begin{cases}(0,0, \xi) & \text { if } a=b=0 \\ (0,1, \xi) & \text { if } a=0 \text { and } b \neq 0 \\ (1, b, \xi) & \text { if } a \neq 0\end{cases}
$$

To unify the presentation of $\Delta(W)$, we make a change of basis:

$$
\begin{aligned}
X & \rightarrow Y, \\
Y & \rightarrow X, \\
Z & \rightarrow-Z, \\
W & \rightarrow W .
\end{aligned}
$$


Under the new basis, the coalgebra structure is the same except for $\Delta(W)$, which becomes

$$
\Delta(W)=1 \otimes W+W \otimes 1+Y \otimes Z-Z \otimes Y+X Y \otimes Y+Y \otimes X Y
$$

The algebraic relations change accordingly, which can be easily done.

From the algebraic relations, $E(a, b, \xi)$ is isomorphic to a universal enveloping algebra of a Lie algebra.

Example 4.6. Let $F$ be the algebra generated by $X, Y, Z, W$ satisfying the following relations:

$$
\begin{aligned}
{[Y, X] } & =[Z, Y]=0, \\
{[Z, X] } & =Y, \\
{[W, X] } & =\beta Y, \\
{[W, Y] } & =\gamma Y, \\
{[W, Z] } & =\gamma Z-\frac{2}{3} Y^{3}+\xi X+\xi^{\prime} Y,
\end{aligned}
$$

where $\beta, \gamma, \xi, \xi^{\prime} \in k$. Then $F$ becomes a bialgebra (and then a Hopf algebra) via

$$
\begin{aligned}
& \epsilon(X)=0, \Delta(X)=1 \otimes X+X \otimes 1, \\
& \epsilon(Y)=0, \Delta(Y)=1 \otimes Y+Y \otimes 1, \\
& \epsilon(Z)=0, \Delta(Z)=1 \otimes Z+X \otimes Y-Y \otimes X+Z \otimes 1, \\
& \epsilon(W)=0, \\
& \begin{aligned}
\Delta(W) & =1 \otimes W+W \otimes 1 \\
& +Y \otimes Z-Z \otimes Y+X Y \otimes Y+Y \otimes X Y .
\end{aligned}
\end{aligned}
$$

If $W$ is replaced by $W_{\text {new }}:=W+\xi^{\prime} X$, then we can assume $\xi^{\prime}=0$. We denote the Hopf algebra by $F(\beta, \gamma, \xi)$. One can make a further reduction by easy linear algebra. For example, if $\gamma \neq 0$, by replacing $X$ by $X_{n e w}:=X-\gamma^{-1} \beta Y$, we have $\beta=0$. By rescaling, we can further assume $\gamma=1$. If $\gamma=0$, then by rescaling, we might assume $\beta=1$. In summary, $\{\beta, \gamma\}$ is either $\{0,1\}$ or $\{1,0\}$. This completely determines the isomorphism classes of the Hopf algebras $F(\beta, \gamma, \xi)$.

Let $W^{\prime}=W-\frac{2}{3} X Y^{2}$. Then the algebraic relations of $F$ become

$$
\begin{aligned}
{[Y, X] } & =[Z, Y]=0, \\
{[Z, X] } & =Y, \\
{\left[W^{\prime}, X\right] } & =\beta Y, \\
{\left[W^{\prime}, Y\right] } & =\gamma Y, \\
{\left[W^{\prime}, Z\right] } & =\gamma Z+\xi X .
\end{aligned}
$$

Therefore the subspace generated by $\left\{X, Y, Z, W^{\prime}\right\}$ is a 4-dimensional Lie algebra, say $\mathfrak{g}$, and $F$ is isomorphic to the enveloping algebra $U(\mathfrak{g})$ as algebras. 
Example 4.7. Let $K$ be the algebra generated by $X, Y, Z, W$ satisfying the following relations:

$$
\begin{aligned}
{[Y, X] } & =[Z, Y]=0, \\
{[Z, X] } & =X, \\
{[W, X] } & =-Z, \\
{[W, Y] } & =0, \\
{[W, Z] } & =W-X Y^{2} .
\end{aligned}
$$

The coalgebra structure of $K$ is given as in (E4.6.1). Then $K$ becomes a Hopf algebra. Let $W^{\prime}=W-\frac{1}{2} X Y^{2}$. Then the algebraic relations become

$$
\begin{aligned}
{[Y, X] } & =[Z, Y]=0, \\
{[Z, X] } & =X, \\
{\left[W^{\prime}, X\right] } & =-Z, \\
{\left[W^{\prime}, Y\right] } & =0, \\
{\left[W^{\prime}, Z\right] } & =W^{\prime} .
\end{aligned}
$$

Therefore the subspace generated by $\left\{X, Y, Z, W^{\prime}\right\}$ is a 4-dimensional Lie algebra, say $\mathfrak{g}$, and $K$ is isomorphic to the enveloping algebra $U(\mathfrak{g})$ as algebras.

Proposition 4.8. The algebras $H$ defined in Examples 4.4 4.7 have the following properties.

(a) $H$ is an iterated Ore extension $k[X]\left[Y ; \delta_{1}\right]\left[Z ; \delta_{2}\right]\left[W ; \sigma_{3}, \delta_{3}\right]$.

(b) $H$ is an Auslander regular Cohen-Macaulay domain.

(c) The global dimension and GK-dimension of $H$ is 4 .

(d) $H$ is a Hopf algebra and connected as a coalgebra.

(e) $P(H)=k X+k Y$. The subalgebra generated by $X, Y$ is the enveloping algebra $U(\mathfrak{g})$ where $\mathfrak{g}$ is the Lie algebra $P(H)$.

(f) $P_{2}(H)=k X+k Y+k Z$. The subalgebra generated by $X, Y, Z$ is the enveloping algebra $U(L)$ where $L$ is the $C L A P_{2}(H)$.

(g) $H$ is not isomorphic to an enveloping algebra of either a Lie algebra or a CLA.

(h) The lantern $\mathfrak{L}(H)$ of $H$ is isomorphic to the graded Lie algebra of dimension 4 , with a basis $\left\{x^{*}, y^{*}, z^{*}, w^{*}\right\}$ such that $z^{*}=\left[x^{*}, y^{*}\right]$ and $w^{*}=\left[z^{*}, y^{*}\right]$, subject to the relation $\left[z^{*}, x^{*}\right]=0=\left[w^{*}, x^{*}\right]=\left[w^{*}, y^{*}\right]$.

Sketch of the proof. (a) Follows from the definitions.

$(b, c)$ These facts are true for any iterated Ore extension.

(d,e,f) These depend on straightforward, but not trivial, computation.

(g) This follows from parts $(\mathrm{e}, \mathrm{f})$.

(h) This was proved in Lemma 1.4(c). We can also check it directly here. Note that $\left\{x^{*}, y^{*}, z^{*}, w^{*}\right\}$ can be viewed as a dual basis of $\{X, Y, Z, W\}$ in gr $H$ and that coproducts of $X, Y, Z, W$ given in Examples 4.4 4.7 match up with the Lie structure of the $\mathfrak{L}(H)$ given in part $(\mathrm{h})$.

4.3. Primitively-thin Hopf algebra of GK-dimension 4, Part I. In this and the next two subsections we classify all primitively-thin Hopf algebras of GKdimension 4 . 
Let $C$ be a primitively-thin Hopf algebra of GK-dimension 3. By Proposition 4.3. $C$ is of type $A$ or $B$ as in Examples 4.1 and 4.2. Let $D$ be the Hopf algebra $A(0,0,0)$. Then it is easy to see that $D$ is a coradically graded Hopf algebra by setting $\operatorname{deg} X=\operatorname{deg} Y=1$ and $\operatorname{deg} Z=2$. Since $\operatorname{gr} C \cong D, C$ is a so-called PBW deformation of $D$.

Let $\{x, y, z\}$ be any set of generators of $C$ such that $x, y$ are primitive and $\Delta(z)=1 \otimes z+x \otimes y-y \otimes x+z \otimes 1$. Then $C$ has a basis consisting of monomials of the form

$$
x^{i_{1}} y^{i_{2}} z^{i_{3}} .
$$

Notice that $C^{+}$is spanned by $x^{i_{1}} y^{i_{2}} z^{i_{3}}$ with at least one $i_{k}$ nonzero. Let $\bar{x}, \bar{y}, \bar{z}$ be the homogeneous elements in $D=\operatorname{gr} C$ corresponding to $x, y, z$, respectively. (In fact, an easy calculation shows that $\bar{x}, \bar{y}, \bar{z}$ can be identified with the canonical generators $X, Y, Z$ as in the definition of $A(0,0,0)$.) Then $\operatorname{gr} C$ has a basis $\left\{\bar{x}^{i_{1}} \bar{y}^{i_{2}} \bar{z}^{i_{3}}\right\}$. Now we have a $k$-space isomorphism from $C$ to gr $C$ by sending $x^{i_{1}} y^{i_{2}} z^{i_{3}}$ to $\bar{x}^{i_{1}} \bar{y}^{i_{2}} \bar{z}^{i_{3}}$. Clearly this isomorphism maps $C^{+}$onto $D^{+}$. From now on we identify $C$ with $D=\operatorname{gr} C$ as $k$-spaces by this isomorphism, and we will abuse the notation by dropping the bars for the generators $\bar{x}, \bar{y}, \bar{z}$ of $\operatorname{gr} C$. Define $\operatorname{deg} x^{i_{1}} y^{i_{2}} z^{i_{3}}=i_{1}+i_{2}+2 i_{3}$. This grading agrees with the natural grading on $D=\operatorname{gr} C$. Moreover, by the defining relations of $C$, it is easy to check that

$$
\Delta_{C}(a)=\Delta_{D}(a)+l d t
$$

where $a \in C$ and $l d t$ denotes terms with degrees lower than $\operatorname{deg} a$. As a consequence, we can think about $\Omega C$ and $\Omega D$, the cobar constructions of $C$ and $D$, as the same graded $k$-spaces with two differentials $\partial_{C}$ and $\partial_{D}$. Moreover, $\partial_{D}$ respects the grading and

where $b \in\left(C^{+}\right)^{\otimes n}$.

$$
\partial_{C}^{n}(b)=\partial_{D}^{n}(b)+l d t
$$

Lemma 4.9. Let $D=A(0,0,0)$. Then $\operatorname{dim}_{k} \mathrm{H}^{2}(\Omega D)=2$ and $\mathrm{H}^{2}(\Omega D)$ is spanned by the classes of 2-cocycles $(u)$ and $(t)$, where

$$
\begin{aligned}
& u=z \otimes x-x \otimes z+x y \otimes x+x \otimes x y, \\
& t=y \otimes z-z \otimes y+x y \otimes y+y \otimes x y .
\end{aligned}
$$

Proof. As mentioned above, $D$ is a graded Hopf algebra. Let $A$ be the graded dual of $D$. Since $D$ is commutative, $A$ is cocommutative. By Proposition 1.1] $A=U(\mathfrak{L})$ for some graded Lie algebra $\mathfrak{L}$. Since $D$ is coradically graded as a coalgebra, $A$ is generated in degree 1 . This implies that $\mathfrak{L}$ is the 3 -dimensional Heisenberg Lie algebra, or equivalently, $A$ is generated by two degree 1 elements $x_{1}$ and $x_{2}$ with relations

$$
x_{1}^{2} x_{2}+x_{2} x_{1}^{2}=2 x_{1} x_{2} x_{1}, \quad x_{2}^{2} x_{1}+x_{1} x_{2}^{2}=2 x_{2} x_{1} x_{2} .
$$

By [LPWZ2, Lemma 8.6 (c)], $B^{\#} A \cong \Omega C$ as DG algebras, where $B^{\#} A$ is the graded dual of the bar construction of $A$. On the other hand, by [LPWZ1, Lemma 4.2], $\mathrm{H}^{\bullet}\left(B^{\#} A\right) \cong \operatorname{Ext}_{A}^{\bullet}\left(k_{A}, k_{A}\right)$. As a consequence,

$$
\operatorname{dim}_{k} \mathrm{H}^{2}(\Omega D)=\operatorname{dim}_{k} \operatorname{Ext}_{A}^{2}\left(k_{A}, k_{A}\right)=2 .
$$

We introduce a $\mathbb{Z}^{2}$-grading on $D$ by setting $\operatorname{deg}_{2} x=(1,0), \operatorname{deg}_{2} y=(0,1)$ and $\operatorname{deg}_{2} z=(1,1)$. Then it is clear that $D$ is a $\mathbb{Z}^{2}$-graded Hopf algebra and therefore the differentials of $\Omega D$ preserve the $\mathbb{Z}^{2}$-grading. A direct computation shows that 
both $u$ and $t$ are 2-cocycles. Now if $u$ is a 2-coboundary, it must be a linear combination of $\partial^{1}\left(x^{2} y\right)=\delta\left(x^{2} y\right)=x^{2} \otimes y+2 x y \otimes x+2 x \otimes x y+y \otimes x^{2}$ and $\partial^{1}(x z)=\delta(x z)=x \otimes z+z \otimes x+x^{2} \otimes y+x \otimes x y$. An easy calculation shows that it is impossible. Hence the class $(u)$ is a nonzero element in $\mathrm{H}^{2}(\Omega D)$. Similarly, one can show that the class $(t)$ is also nonzero in $\mathrm{H}^{2}(\Omega D)$. Moreover, they are linearly independent since they have different $\mathbb{Z}^{2}$-degrees. This completes the proof.

Recall that monomials of the form $x^{i} y^{j} z^{k}$ in $C$ are identified with monomials $x^{i} y^{j} z^{k}$ in $D$.

Proposition 4.10. Retain the above notation. Then $\mathrm{H}^{2}(\Omega C)$ is spanned by the classes of 2-cocycles $(u)$ and $(t)$, where $u$ and $t$ are given as in (E4.9.1)-(E4.9.2), and $\operatorname{dim}_{k} \mathrm{H}^{2}(\Omega C)=2$.

Proof. Let $w$ be a nonzero linear combination of $u$ and $t$. Then $w$ is homogeneous of degree 3 and $\partial_{C}^{2}(w)=\partial_{D}^{2}(w)=0$ by direct calculation (which only uses (E4.1.1)). Suppose that there exists $c \in C^{+}$such that $w=\partial_{C}^{1}(c)$. Also, we can write $\partial_{C}^{1}(c)=$ $\partial_{D}^{1}(c)+v$, where $v \in\left(C^{+}\right)^{\otimes 2}$ has degree less than the degree of $c$. If the degree of $c$ is larger than 3 , then $\operatorname{deg} \partial_{D}^{1}(c)=\operatorname{deg} c>3$ as $\partial_{D}^{1}$ is homogeneous and $\partial_{D}^{1}(c) \neq 0$. Then

$$
\operatorname{deg} w=\operatorname{deg} \partial_{C}^{1}(c)=\operatorname{deg} \partial_{D}^{1}(c)>3,
$$

a contradiction. Therefore $\operatorname{deg} c \leq 3$ and consequently, $v$ has degree less than 3 .

Since $\partial_{D}^{2} \partial_{C}^{1}(c)=\partial_{D}^{2}(w)=0$, we have $\partial_{D}^{2}\left(\partial_{D}^{1}(c)+v\right)=0$. Hence $\partial_{D}^{2}(v)=0$. But $\operatorname{deg} v<3$, so by Lemma 4.9, there exists $c^{\prime} \in C^{+}$such that $v=\partial_{D}^{1}\left(c^{\prime}\right)$. As a consequence, $w=\partial_{D}^{1}(c)+v=\partial_{D}^{1}\left(c+c^{\prime}\right)$, which is a contradiction.

Now, we have shown that $\mathrm{H}^{2}(\Omega C)$ is at least of dimension 2 and $(u),(t)$ are linearly independent in $\mathrm{H}^{2}(\Omega C)$. On the other hand, by a standard spectral sequence argument [We, Theorem 5.5.1], we have $\operatorname{dim}_{k} \mathrm{H}^{2}(\Omega C) \leq \operatorname{dim}_{k} \mathrm{H}^{2}(\Omega D)=2$. This completes the proof.

Lemma 4.11. Let $H$ be a connected coalgebra and $K$ a proper subcoalgebra of $H$. Let $N$ be the smallest number such that $K_{N} \subsetneq H_{N}$ and suppose that $N \geq 2$; then $\delta$ induces an injective $k$-linear map from $H_{N}^{+} / K_{N}^{+}$to $\mathrm{H}^{2}(\Omega K)$.

Proof. By the choice of $N$, we see that, for any $g \in H_{N}^{+}$,

$$
\delta(g)=\Delta(g)-(1 \otimes g+g \otimes 1) \in H_{N-1}^{+} \otimes H_{N-1}^{+}=K_{N-1}^{+} \otimes K_{N-1}^{+} .
$$

Hence $\partial_{K}^{2}(\delta(g))=\partial_{K}^{2} \partial_{H}^{1}(g)=\partial_{H}^{2} \partial_{H}^{1}(g)=0$, which means that $\delta(g)$ is a 2-cocycle in the complex $\Omega K$. Hence $\delta$ defines a $k$-linear map from $H_{N}^{+}$to $\mathrm{H}^{2}(\Omega K)$. For any element $g \in K_{N}^{+}, \delta(g)=\partial_{K}^{1}(g)$ is a 2-boundary in the complex $\Omega K$, whence it is zero in $\mathrm{H}^{2}(\Omega K)$. Thus $\delta$ induces a $k$-linear map from $H_{N}^{+} / K_{N}^{+}$to $\mathrm{H}^{2}(\Omega K)$.

If $g \in H_{N}^{+} \backslash K_{N}^{+}$, we claim that $\delta(g)$ represents a nonzero cohomology class in $\mathrm{H}^{2}(\Omega K)$. If not, there is $w \in K^{+}$such that $\partial_{K}^{1}(w)=\delta(w)=\delta(g)$. As a consequence, $\Delta(g-w)=1 \otimes(g-w)+(g-w) \otimes 1$; i.e. $g-w$ is a primitive element in $H$. By the fact that $H_{1}^{+}=K_{1}^{+}, g-w \in K_{1}^{+}$. But this would imply that $g \in K^{+}$, which contradicts the choice of $g$. Therefore the map from $H_{N}^{+} / K_{N}^{+}$to $\mathrm{H}^{2}(\Omega K)$ is injective.

Theorem 4.12. Suppose that $H$ is a primitively-thin Hopf algebra of GK-dimension 4. Then for any linearly independent primitive elements $x, y$, there exists $z \in H$ 
such that

$$
\Delta(z)=1 \otimes z+x \otimes y-y \otimes x+z \otimes 1 .
$$

For any such $z$, the algebra $C$ generated by $\{x, y, z\}$ is a Hopf subalgebra of $G K$ dimension 3. Moreover, there exists $w \in H$ such that

$$
\Delta(w)=1 \otimes w+\theta_{1} u+\theta_{2} t+w \otimes 1,
$$

where $u$ and $t$ are given in (E4.9.1) For any such $w$, the set $\{x, y, z, w\}$ generates $H$.

Proof. By [Zh2, Prop. 7.10], we can find a Hopf subalgebra $C$ of GK-dimension 3. By Proposition 4.3, there is a $z \in C$ such that $\Delta(z)$ is of the form (E4.12.1). Since $P(C)=k x \oplus k y=P(H), C_{1}=H_{1}$.

Let $N \geq 2$ be the smallest integer such that $C_{N} \subsetneq H_{N}$. By $\underline{\mathrm{Mo}}$, Lemma 5.3.2], there exists $w^{\prime} \in H_{N} \backslash C_{N}$ such that $\Delta\left(w^{\prime}\right)=1 \otimes w^{\prime}+w^{\prime} \otimes 1+f$ where $f \in C_{N-1} \otimes C_{N-1}$. Without loss of generality, we assume that $w^{\prime} \in H^{+}$.

By Lemma 4.11, $f$ represents a nonzero cohomology class in $\mathrm{H}^{2}(\Omega C)$. By Proposition 4.10, the cohomology classes in $\mathrm{H}^{2}(\Omega C)$ represented by $f$ form a nonzero linear combination of $(u)$ and $(t)$. Hence there exist $v \in C^{+}$and $\theta_{1}, \theta_{2} \in k$ such that $f=\partial^{1}(v)+\theta_{1} u+\theta_{2} t$, where at least one of $\theta_{i}$ is nonzero. Let $w=w^{\prime}+v$. Then $w \notin C$ and $\Delta(w)=1 \otimes w+\theta_{1} u+\theta_{2} t+w \otimes 1$.

Next we have to show that $H$ is generated by $x, y, z$ and $w$. Let $K$ be the subalgebra of $H$ generated by $x, y, z$ and $w$. Then it is easy to check that $K$ is a subbialgebra and thus a Hopf subalgebra of $H$. By the construction of $K, C \subsetneq K$. By [Zh2, Lemma 6.8], GKdimgr $K \geq \operatorname{GKdimgr} C+1=4$. On the other hand, GKdim gr $K=\operatorname{GKdim} K \leq \operatorname{GKdim} H=4$ since $K \subset H$. Hence GKdim $K=4$. Now it follows from [Zh2, Lemma 7.4] that $K=H$. This completes the proof.

As a direct consequence, we have the following corollary.

Corollary 4.13. Let $H$ be a commutative, connected, primitively-thin Hopf algebra of GK-dimension 4. Then $H$ is isomorphic to $D(\{0,1\},\{0\},\{0\})$.

Proof. By Theorem 4.12, there is a surjective Hopf map from $D\left(\left\{\theta_{i}\right\},\{0\},\{0\}\right)$ to $H$ sending $X, Y, Z, W$ to $x, y, z, w$, respectively, for some $\left\{\theta_{1}, \theta_{2}\right\}$. The map must be an isomorphism since both $D$ and $H$ are domains of GK-dimension 4 . By definition, $D\left(\left\{\theta_{i}\right\},\{0\},\{0\}\right)$ is a graded Hopf algebra with $\operatorname{deg} X=\operatorname{deg} Y=1, \operatorname{deg} Z=2$ and $\operatorname{deg} W=3$. Hence the graded dual $H^{*}$ is a graded commutative Hopf algebra, which must be isomorphic to the enveloping algebra $U(\mathfrak{L})$ for some graded Lie algebra generated by two elements in degree 1 . Such a Lie algebra is unique (up to isomorphism) and is given in Proposition 4.8(h). Therefore $H$ is isomorphic to $U(\mathfrak{L})^{*}$, which is isomorphic to $D(\{0,1\},\{0\},\{0\})$.

Lemma 4.14. Retain the notation in Theorem 4.12 for parts $(\mathrm{b}, \mathrm{c})$.

(a) The Hopf algebra $D:=D(\{0,1\},\{0\},\{0\})$ is coradically graded by setting $\operatorname{deg} X=\operatorname{deg} Y=1, \operatorname{deg} Z=2$ and $\operatorname{deg} W=3$.

(b) For any connected Hopf algebra $H$ of $G K$-dimension 4 with $\operatorname{dim}_{k} P(H)=2$, gr $H$ is isomorphic to $D(\{0,1\},\{0\},\{0\})$.

(c) Working with gr $C$ (isomorphic to $A(0,0,0)$ ) and gr $H$, we have $C_{2}=H_{2}$ and $\mathrm{H}_{3}^{+} / \mathrm{C}_{3}^{+}$is 1-dimensional, which is spanned by the image of $w$. 
Proof. Let $D$ denote the Hopf algebra $D(\{0,1\},\{0\},\{0\})$.

(a) One can check directly that gr $D=D$. Hence $D$ is coradically graded.

(b) By [Zh2, Theorem 1.2], gr $H$ is commutative, and it is still connected and primitively-thin. The assertion follows from Corollary 4.13

(c) We may replace gr $C$ by $A(0,0,0)$ and gr $H$ by $D$. Then the assertion follows by an easy computation.

Proposition 4.15. Retain the notation in Theorem 4.12. Then $H$ has a $k$-basis of the form

$$
\left\{x^{i_{1}} y^{i_{2}} z^{i_{3}} w^{i_{4}} \mid i_{1}, i_{2}, i_{3}, i_{4} \geq 0\right\} .
$$

Proof. Let $X, Y, Z, W$ be the elements in gr $H$ corresponding to elements $x, y, z, w$ in $H$. Then $\{X, Y, Z, W\}$ generates gr $H$ as an algebra by Theorem 4.12. By Lemma 4.14(b), gr $H \cong D$, so $X, Y, Z, W$ satisfy the defining relations of $D(\{0,1\},\{0\},\{0\})$ given in Example 4.4. As a consequence, gr $H$ has a $k$-basis of the form

$$
\left\{X^{i_{1}} Y^{i_{2}} Z^{i_{3}} W^{i_{4}} \mid i_{1}, i_{2}, i_{3}, i_{4} \geq 0\right\} .
$$

Now the result follows.

In Theorem 4.12, the Hopf subalgebra $C$ is primitively-thin. Hence by Proposition 4.3. $C$ must be isomorphic to either $A\left(\lambda_{1}, \lambda_{2}, \alpha\right)$ or $B(\lambda)$.

4.4. Primitively-thin Hopf algebra of GK-dimension 4, Part II. In this subsection we show that $B(\lambda)$ cannot appear as a Hopf subalgebra of a primitivelythin Hopf algebra of GK-dimension 4. We start with an easy observation.

Lemma 4.16. Let $x$ and $y$ be primitive elements. Then

$$
\begin{gathered}
\delta\left(x y^{2}\right)=y^{2} \otimes x+x \otimes y^{2}+2(x y \otimes y+y \otimes x y), \\
\delta\left(x^{2} y\right)=y \otimes x^{2}+x^{2} \otimes y+2(x y \otimes x+x \otimes x y), \\
\delta\left(y^{3}\right)=3\left(y \otimes y^{2}+y^{2} \otimes y\right) .
\end{gathered}
$$

Proposition 4.17. Retain the notation in Theorem 4.12, Then the Hopf subalgebra $C$ cannot be isomorphic to $B(\lambda)$.

Proof. Suppose to the contrary that $C$ is isomorphic to $B(\lambda)$ for some $\lambda \in k$. Therefore we can assume that $x, y, z \in C$ satisfies the relations listed in Example 4.2 .

First we assume that $\theta_{1}$ is not zero. By dividing $w$ with $\theta_{1}$ we may assume that $\Delta(w)=1 \otimes w+u+\theta_{2} t+w \otimes 1$. Using (E4.12.2), (E4.16.1), (E4.16.3) and the relations of $B(\lambda)$, we have

$$
\begin{aligned}
\delta([w, y])= & \Delta([w, y])-[w, y] \otimes 1-1 \otimes[w, y] \\
= & {[\Delta(w), \Delta(y)]-[w, y] \otimes 1-1 \otimes[w, y] } \\
= & z \otimes[x, y]-[x, y] \otimes z \\
& +[x y, y] \otimes x+x \otimes[x y, y]+x y \otimes[x, y]+[x, y] \otimes x y \\
& +\theta_{2}([x y, y] \otimes y+y \otimes[x y, y]) \\
= & -t+\delta\left(x y^{2}\right)+\frac{\theta_{2}}{3} \delta\left(y^{3}\right) .
\end{aligned}
$$


Let $w^{\prime}=x y^{2}+\frac{\theta_{2}}{3} y^{3}-[w, y]$, then $\delta\left(w^{\prime}\right)=t$, and, whence,

$$
\Delta\left(w^{\prime}\right)=1 \otimes w^{\prime}+t+w^{\prime} \otimes 1 .
$$

Now, under the map given in Lemma 4.11, the elements $w$ and $w^{\prime}$ are mapped to $(u)+\theta_{2}(t)$ and $(t)$, respectively. Hence $\operatorname{dim}_{k} H_{3}^{+} / C_{3}^{+}=2$, which contradicts Lemma 4.14 (c).

Next we assume that $\theta_{1}=0$ and $\theta_{2} \neq 0$. By dividing $w$ with $\theta_{2}$ we may assume that $\Delta(w)=1 \otimes w+t+w \otimes 1$. A similar calculation shows that $[w, x]+2 w \in P(H)$ and $[w, y]-\frac{1}{3} y^{3} \in P(H)$. As a consequence,

$$
\begin{aligned}
\delta([w, z])= & {[w, x] \otimes y-y \otimes[w, x]+x \otimes[w, y]-[w, y] \otimes x } \\
& +[y, z] \otimes z-z \otimes[y, z]+[x y, z] \otimes y+y \otimes[x y, z] \\
& +[t, x \otimes y-y \otimes x] \\
= & -2 w \otimes y+2 y \otimes w+\sum_{s} \alpha_{s} f_{s} \otimes g_{s},
\end{aligned}
$$

where $\alpha_{s} \in k$ and $f_{s}, g_{s}$ are monomials of the form $x^{i_{1}} y^{i_{2}} z^{i_{3}}$. Let gr $H(n)$ denote the degree $n$ piece of the graded Hopf algebra gr $H$. Since gr $H$ is commutative, $[w, z]$ represents an element $V \in \operatorname{gr} H(4)$. Let $X \in \operatorname{gr} H(1), Y \in \operatorname{gr} H(1), Z \in \operatorname{gr} H(2)$ and $W \in$ gr $H(3)$ be the homogeneous elements in gr $H$ corresponding to elements $x, y, z$ and $w$ in $H$, respectively. By (E4.17.1), we see that

$$
\delta(V)=-2 W \otimes Y+2 Y \otimes W+\sum_{s} \alpha_{s}^{\prime} f_{s}^{\prime} \otimes g_{s}^{\prime}
$$

where $\alpha_{s}^{\prime} \in k$ and $f_{s}^{\prime}, g_{s}^{\prime}$ are monomials of the form $X^{i_{1}} Y^{i_{2}} Z^{i_{3}}$.

Now by Theorem 4.12 the set $\{X, Y, Z, W\}$ generates gr $H$. Also, gr $H$ is a $\mathbb{Z}^{2}$-graded coalgebra by setting $\operatorname{deg}_{2} X=(1,0), \operatorname{deg}_{2} Y=(0,1), \operatorname{deg}_{2} Z=(1,1)$ and $\operatorname{deg}_{2} W=(1,2)$. Notice that $\operatorname{deg}_{2} V=(1,3)$. Therefore, $V$ must be a linear combination of linearly independent elements $X Y^{3}, Y^{2} Z, Y W$ of degree $(1,3)$. Hence there are $\beta_{i} \in k$ such that

$$
V=\beta_{1} X Y^{3}+\beta_{2} Z Y^{2}+\beta_{3} Y W
$$

or

$$
\begin{aligned}
\delta(V) & =\delta\left(\beta_{1} X Y^{3}+\beta_{2} Z Y^{2}+\beta_{3} Y W\right) \\
& =\beta_{3}(Y \otimes W+W \otimes Y)+\sum_{s} \alpha_{s}^{\prime \prime} f_{s}^{\prime \prime} \otimes g_{s}^{\prime \prime},
\end{aligned}
$$

where $\alpha_{s}^{\prime \prime} \in k$ and $f_{s}^{\prime \prime}, g_{s}^{\prime \prime}$ are monomials of the form $X^{i_{1}} Y^{i_{2}} Z^{i_{3}}$. If we compare the coefficient of $W \otimes Y$ on (E4.17.2) and (E4.17.3), we get $-2=\beta_{3}$. On the other hand, if we compare the coefficient of $Y \otimes W$, we have $2=\beta_{3}$, which is a contradiction. This completes the proof.

4.5. Primitively-thin Hopf algebra of GK-dimension 4, Part III. In this subsection we deal with the case when $C=A\left(\lambda_{1}, \lambda_{2}, \alpha\right)$ and finish the analysis. Throughout this subsection we assume that $C=A\left(\lambda_{1}, \lambda_{2}, \alpha\right)$ where the relations of $A\left(\lambda_{1}, \lambda_{2}, \alpha\right)$ are given in Example 4.1 and that $\left(\lambda_{1}, \lambda_{2}, \alpha\right)$ is either $(0,0,0)$ or $(0,0,1)$ or $(1,1,1)$ or $(1, \lambda, 0)$ as listed in Proposition 4.3(a). 
Lemma 4.18. Let $u$ and $t$ be given as in (E4.9.1) (E4.9.2).

$$
\begin{aligned}
{[u, x \otimes 1+1 \otimes x] } & =\alpha(y \otimes x-x \otimes y), \\
{[t, x \otimes 1+1 \otimes x] } & =\lambda_{1}(y \otimes x-x \otimes y), \\
{[u, y \otimes 1+1 \otimes y] } & =\lambda_{2}(y \otimes x-x \otimes y), \\
{[t, y \otimes 1+1 \otimes y] } & =0 .
\end{aligned}
$$

Proof. We use the relations of $A\left(\lambda_{1}, \lambda_{2}, \alpha\right)$ and note that $[x, y]=0$. By an easy computation, we have

$$
\begin{aligned}
& {[u, x \otimes 1]=[z, x] \otimes x=\left(\lambda_{1} x+\alpha y\right) \otimes x,} \\
& {[u, 1 \otimes x]=-x \otimes[z, x]=-x \otimes\left(\lambda_{1} x+\alpha y\right) .}
\end{aligned}
$$

Now (E4.18.1) follows by adding the above.

By a computation,

$$
\begin{aligned}
& {[t, x \otimes 1]=-[z, x] \otimes y=-\left(\lambda_{1} x+\alpha y\right) \otimes y,} \\
& {[t, 1 \otimes x]=y \otimes[z, x]=y \otimes\left(\lambda_{1} x+\alpha y\right),}
\end{aligned}
$$

and (E4.18.2) is obtained by adding the above. The proofs of the last two are similar.

Lemma 4.19. Let $w$ be as in Theorem 4.12.

$$
\begin{aligned}
& {[w, x]=-\left(\theta_{1} \alpha+\theta_{2} \lambda_{1}\right) z+a_{11} x+a_{12} y,} \\
& {[w, y]=-\theta_{1} \lambda_{2} z+a_{21} x+a_{22} y}
\end{aligned}
$$

for some $a_{11}, a_{12}, a_{21}, a_{22} \in k$.

Proof. We only prove the first equation; the proof of the second equation is similar.

$$
\begin{aligned}
\delta([w, x]) & =\Delta([w, x])-[w, x] \otimes 1-1 \otimes[w, x] \\
& =[\Delta(w), x \otimes 1+1 \otimes x]-[w, x] \otimes 1-1 \otimes[w, x] \\
& =\left[w \otimes 1+1 \otimes w+\theta_{1} u+\theta_{2} t, x \otimes 1+1 \otimes x\right]-[w, x] \otimes 1-1 \otimes[w, x] \\
& =\left[\theta_{1} u+\theta_{2} t, x \otimes 1+1 \otimes x\right] \\
& =\theta_{1}[u, x \otimes 1+1 \otimes x]+\theta_{2}[t, x \otimes 1+1 \otimes x] \\
& =\left(\theta_{1} \alpha+\theta_{2} \lambda_{1}\right)(y \otimes x-x \otimes y) \quad \text { by (E4.18.1)-(E4.18.2) } \\
& =-\left(\theta_{1} \alpha+\theta_{2} \lambda_{1}\right) \delta(z) .
\end{aligned}
$$

Therefore $[w, x]+\left(\theta_{1} \alpha+\theta_{2} \lambda_{1}\right) z$ is a primitive element, whence, it is of the form $a_{11} x+a_{12} y$ for some $a_{11}, a_{12} \in k$. The assertion follows.

Lemma 4.20. Retain the notation as above. Then $\lambda_{2}=0$. Consequently $\left(\lambda_{1}, \lambda_{2}, \alpha\right)$ is either $(0,0,0)$ or $(0,0,1)$ or $(1,0,0)$.

Proof. Since $[x, y]=0$, using Lemma 4.19 we have

$$
\begin{aligned}
0 & =[w,[x, y]]=[[w, x], y]+[x,[w, y]] \\
& =\left[-\left(\theta_{1} \alpha+\theta_{2} \lambda_{1}\right) z+a_{11} x+a_{12} y, y\right]+\left[x,-\theta_{1} \lambda_{2} z+a_{21} x+a_{22} y\right] \\
& =-\left(\theta_{1} \alpha+\theta_{2} \lambda_{1}\right)[z, y]+\theta_{1} \lambda_{2}[z, x] \\
& =-\left(\theta_{1} \alpha+\theta_{2} \lambda_{1}\right)\left(\lambda_{2} y\right)+\theta_{1} \lambda_{2}\left(\lambda_{1} x+\alpha y\right) \\
& =-\theta_{2} \lambda_{1} \lambda_{2} y+\theta_{1} \lambda_{1} \lambda_{2} x .
\end{aligned}
$$


Since one of the $\theta_{i}$ 's is nonzero, $\lambda_{1} \lambda_{2}=0$. We only consider those $\left(\lambda_{1}, \lambda_{2}, \alpha\right)$ 's given in Proposition 4.3(a); therefore, $\lambda_{2}=0$.

Lemma 4.21. Suppose $\lambda_{2}=0$. Let $u$ and $t$ be given as in (E4.9.1)-(E4.9.2).

$$
\begin{aligned}
& {[u, z \otimes 1+1 \otimes z]=-\lambda_{1} u+\alpha t-\alpha \delta\left(x y^{2}\right)-\lambda_{1}(x y \otimes x+x \otimes x y),} \\
& (\mathrm{E} 4.21 .2) \quad[t, z \otimes 1+1 \otimes z]=-\lambda_{1}(x y \otimes y+y \otimes x y)-\alpha\left(y^{2} \otimes y+y \otimes y^{2}\right), \\
& (\mathrm{E} 4.21 .3) \quad[u, x \otimes y-y \otimes x]=\lambda_{1}(x \otimes x y+x y \otimes x)+\alpha(y \otimes x y+x y \otimes y) \text {, } \\
& (\mathrm{E} 4.21 .4) \quad[t, x \otimes y-y \otimes x]=-\lambda_{1}\left(x \otimes y^{2}+y^{2} \otimes x\right)-\alpha\left(y \otimes y^{2}+y^{2} \otimes y\right) .
\end{aligned}
$$

Proof. By a direct computation, we have

$$
\begin{aligned}
& {[u, z \otimes 1]=\left(\lambda_{1} x+\alpha y\right) \otimes z-\left(\lambda_{1} x+\alpha y\right) y \otimes x-\left(\lambda_{1} x+\alpha y\right) \otimes x y,} \\
& {[u, 1 \otimes z]=-z \otimes\left(\lambda_{1} x+\alpha y\right)-x y \otimes\left(\lambda_{1} x+\alpha y\right)-x \otimes\left(\lambda_{1} x+\alpha y\right) y .}
\end{aligned}
$$

Adding up and using definitions and (E4.16.1), we obtain (E4.21.1). Others are similar by using definitions and direct computations.

Lemma 4.22. Suppose $\lambda_{2}=0$. Then

$$
\theta_{1}\left(\theta_{2} \lambda_{1}+\theta_{1} \alpha\right)=0 \text {. }
$$

Further,

(a) If $\left(\lambda_{1}, \lambda_{2}, \alpha\right)=(0,0,0)$, then

$$
[w, z]=\left(a_{11}+a_{22}\right) z+\xi_{1} x+\xi_{2} y
$$

for some $\xi_{i} \in k$

(b) If $\left(\lambda_{1}, \lambda_{2}, \alpha\right)=(1,0,0)$, then $\theta_{1} \theta_{2}=0$. If, moreover, $\theta_{1}=0$, then

$$
[w, z]=\left(a_{11}+a_{22}\right) z+w+\left(-\theta_{2}\right) x y^{2}+\xi_{1} x+\xi_{2} y
$$

for some $\xi_{i} \in k$. If, moreover, $\theta_{2}=0$, then

$$
[w, z]=\left(a_{11}+a_{22}\right) z-w+\xi_{1} x+\xi_{2} y
$$

for some $\xi_{i} \in k$.

(c) If $\left(\lambda_{1}, \lambda_{2}, \alpha\right)=(0,0,1)$, then $\theta_{1}=0$ and

$$
[w, z]=\left(a_{11}+a_{22}\right) z-\frac{2}{3} \theta_{2} y^{3}+\xi_{1} x+\xi_{2} y
$$

for some $\xi_{i} \in k$. 
Proof.

$$
\begin{aligned}
\delta([w, z])= & \Delta([w, z])-[w, z] \otimes 1-1 \otimes[w, z] \\
= & {[\Delta(w), \Delta(z)]-[w, z] \otimes 1-1 \otimes[w, z] } \\
= & {\left[w \otimes 1+1 \otimes w+\theta_{1} u+\theta_{2} t, z \otimes 1+1 \otimes z+(x \otimes y-y \otimes x)\right] } \\
& -[w, z] \otimes 1-1 \otimes[w, z] \\
= & {[w \otimes 1+1 \otimes w,(x \otimes y-y \otimes x)] } \\
& +\left[\theta_{1} u+\theta_{2} t, z \otimes 1+1 \otimes z+(x \otimes y-y \otimes x)\right] \\
= & -\left(\theta_{1} \alpha+\theta_{2} \lambda_{1}\right)(z \otimes y-y \otimes z)+\left(a_{11}+a_{22}\right)(x \otimes y-y \otimes x) \\
& +\theta_{1}\left(-\lambda_{1} u+\alpha t-\alpha \delta\left(x y^{2}\right)+\alpha(y \otimes x y+x y \otimes y)\right) \\
& +\theta_{2}\left(-\lambda_{1} \delta\left(x y^{2}\right)+\lambda_{1}(x y \otimes y+y \otimes x y)-2 \alpha\left(y^{2} \otimes y+y \otimes y^{2}\right)\right) \\
& \quad \text { by (E4.19.1)-(E4.19.2) } \\
= & \theta_{1} \lambda_{1} u+\left(2 \theta_{1} \alpha+\theta_{2} \lambda_{1}\right) t \\
& +\left(a_{11}+a_{22}\right) \delta(z)+\left(-\theta_{1} \alpha-\theta_{2} \lambda_{1}\right) \delta\left(x y^{2}\right)-\frac{2}{3} \theta_{2} \alpha \delta\left(y^{3}\right) .
\end{aligned}
$$

Since $\delta([w, z]) \in C \otimes C,[w, z]$ induces a cohomology class in $\mathrm{H}^{2}(\Omega C)$. By Lemma $4.14(c), \delta([w, z])$ is a scalar multiple of $\delta(w)$. This implies that

$$
\theta_{2}\left(-\theta_{1} \lambda_{1}\right)-\theta_{1}\left(2 \theta_{1} \alpha+\theta_{2} \lambda_{1}\right)=0
$$

or, after simplifying, we obtain (E4.22.1).

(a) By the above computation, we have $\delta([w, z])=\left(a_{11}+a_{22}\right) \delta(z)$. The assertion follows.

(b) When $\left(\lambda_{1}, \lambda_{2}, \alpha\right)=(1,0,0)$, E4.22.1) becomes $\theta_{1} \theta_{2}=0$ and

$$
\delta([w, z])=-\theta_{1} u+\theta_{2} t+\left(a_{11}+a_{22}\right) \delta(z)-\theta_{2} \delta\left(x y^{2}\right) .
$$

Since $\theta_{1} \theta_{2}=0,-\theta_{1} u+\theta_{2} t$ is either $\delta(w)$ or $-\delta(w)$. Hence we have

$$
[w, z]=\left(a_{11}+a_{22}\right) z+c w+\left(-\theta_{2}\right) x y^{2}+\xi_{1} x+\xi_{2} y
$$

for some $\xi_{i} \in k$ and $c= \pm 1$, which gives the two cases listed in part (b).

(c) If $\left(\lambda_{1}, \lambda_{2}, \alpha\right)=(0,0,1)$, then (E4.22.1) implies that $\theta_{1}=0$ and

$$
\delta([w, z])=\left(a_{11}+a_{22}\right) \delta(z)-\frac{2}{3} \theta_{2} \delta\left(y^{3}\right),
$$

and therefore the assertion follows.

Theorem 4.23. Let $H$ be a connected Hopf algebra of GK-dimension 4 with $\operatorname{dim}_{k} P(H)=2$. Then $H$ must be isomorphic to one of the Hopf algebras listed in Examples 4.4, 4.5, 4.6 and 4.7.

Proof. Retain the notation in Theorem 4.12, By Proposition 4.17 and Proposition 4.3. $C$ must be isomorphic to $A\left(\lambda_{1}, \lambda_{2}, \alpha\right)$, where the possible choices of $\left(\lambda_{1}, \lambda_{2}, \alpha\right)$ are listed in Proposition 4.3(a). By Lemma 4.20, $\lambda_{2}=0$. Hence $C$ is either $A(0,0,0)$ or $A(1,0,0)$ or $A(0,0,1)$. 
Case 1: $C=A(0,0,0)$. By Lemma 4.19 and 4.22(a), we have

$$
\begin{aligned}
& {[w, x]=a_{11} x+a_{12} y,} \\
& {[w, y]=a_{21} x+a_{22} y,} \\
& {[w, z]=\left(a_{11}+a_{22}\right) z+\xi_{1} x+\xi_{2} y}
\end{aligned}
$$

for some $a_{11}, a_{12}, a_{21}, a_{22}, \xi_{1}, \xi_{2} \in k$. Together with the coalgebra given in Theorem 4.12. this is the Hopf algebra described in Example 4.4.

Case 2: $C=A(1,0,0)$. By Lemma 4.22(b), there are two cases to consider. If $\theta_{1}=0$, by dividing $w$ with $\theta_{2}$ we can assume that $\theta_{2}=1$. In this setting, by Lemmas 4.19 and 4.22 (b), we have

$$
\begin{aligned}
& {[w, x]=-z+a_{11} x+a_{12} y} \\
& {[w, y]=a_{21} x+a_{22} y} \\
& {[w, z]=\left(a_{11}+a_{22}\right) z+w-x y^{2}+\xi_{1} x+\xi_{2} y,}
\end{aligned}
$$

for some $a_{11}, a_{12}, a_{21}, a_{22}, \xi_{1}, \xi_{2} \in k$. Applying $[w,-]$ to $[z, y]=0$, one sees that

$$
\begin{aligned}
0 & =[w,[z, y]]=[[w, z], y]+[z,[w, y]] \\
& =\left[\left(a_{11}+a_{22}\right) z+w-x y^{2}+\xi_{1} x+\xi_{2} y, y\right]+\left[z, a_{21} x+a_{22} y\right] \\
& =[w, y]+a_{21}[z, x]=a_{21} x+a_{22} y+a_{21} x .
\end{aligned}
$$

Thus $a_{21}=a_{22}=0$. Applying $[w,-]$ to $[z, x]=x$ and using $a_{21}=a_{22}=0$, one sees that

$$
\begin{aligned}
{[w, x] } & =[w,[z, x]] \\
& =[[w, z], x]+[z,[w, x]] \\
& =\left[a_{11} z+w-x y^{2}+\xi_{1} x+\xi_{2} y, x\right]+\left[z,-z+a_{11} x+a_{12} y\right] \\
& =a_{11}[z, x]+[w, x]+a_{11}[z, x]=[w, x]+2 a_{11} x
\end{aligned}
$$

which implies that $a_{11}=0$. By setting $z_{\text {new }}=z-a_{12} y$, we can make $a_{12}=0$. By setting $w_{\text {new }}=w+\frac{1}{2} \xi_{1} x+\xi_{2} y$, we can make $\xi_{1}=\xi_{2}=0$. New variables $z$ and $w$ still satisfy (E4.12.1) and (E4.12.2), respectively (for $\theta_{1}=0$ ). Therefore this is the Hopf algebra described in Example 4.7.

If $\theta_{2}=0$, by dividing $w$ with $\theta_{1}$ we can assume that $\theta_{1}=1$. In this setting, by Lemmas 4.19 and 4.22 (b), we have

$$
\begin{aligned}
& {[w, x]=a_{11} x+a_{12} y,} \\
& {[w, y]=a_{21} x+a_{22} y,} \\
& {[w, z]=\left(a_{11}+a_{22}\right) z-w+\xi_{1} x+\xi_{2} y}
\end{aligned}
$$

for some $a_{11}, a_{12}, a_{21}, a_{22}, \xi_{1}, \xi_{2} \in k$. Applying $[w,-]$ to $[z, y]=0$, one sees that

$$
\begin{aligned}
0 & =[w,[z, y]]=[[w, z], y]+[z,[w, y]] \\
& =\left[\left(a_{11}+a_{22}\right) z-w+\xi_{1} x+\xi_{2} y, y\right]+\left[z, a_{21} x+a_{22} y\right] \\
& =-[w, y]+a_{21}[z, x]=-a_{22} y .
\end{aligned}
$$


Thus $a_{22}=0$. Applying $[w,-]$ to $[z, x]=x$ and using $a_{22}=0$, one sees that

$$
\begin{aligned}
{[w, x] } & =[w,[z, x]] \\
& =[[w, z], x]+[z,[w, x]] \\
& =\left[a_{11} z-w+\xi_{1} x+\xi_{2} y, x\right]+\left[z, a_{11} x+a_{12} y\right] \\
& =a_{11}[z, x]-[w, x]+a_{11}[z, x]=-[w, x]+2 a_{11} x,
\end{aligned}
$$

which implies that $a_{12}=0$. This is the Hopf algebra described in Example 4.5.

Case 3: $C=A(0,0,1)$. By Lemma 4.22 (c), $\theta_{1}=0$. By dividing $w$ by $\theta_{2}$ we can assume that $\theta_{2}=1$. In this setting, by Lemmas 4.19 and 4.22 (c), we have

$$
\begin{aligned}
& {[w, x]=a_{11} x+a_{12} y,} \\
& {[w, y]=a_{21} x+a_{22} y,} \\
& {[w, z]=\left(a_{11}+a_{22}\right) z-\frac{2}{3} y^{3}+\xi_{1} x+\xi_{2} y,}
\end{aligned}
$$

for some $a_{11}, a_{12}, a_{21}, a_{22}, \xi_{1}, \xi_{2} \in k$. Applying $[w,-]$ to $[z, y]=0$, one sees that

$$
\begin{aligned}
0 & =[w,[z, y]]=[[w, z], y]+[z,[w, y]] \\
& =\left[\left(a_{11}+a_{22}\right) z-\frac{2}{3} y^{3}+\xi_{1} x+\xi_{2} y, y\right]+\left[z, a_{21} x+a_{22} y\right] \\
& =a_{21} y .
\end{aligned}
$$

Hence $a_{21}=0$. Applying $[w,-]$ to $[z, x]=y$, one sees that

$$
\begin{aligned}
a_{22} y & =[w, y]=[w,[z, x]]=[[w, z], x]+[z,[w, x]] \\
& =\left[\left(a_{11}+a_{22}\right) z-\frac{2}{3} y^{3}+\xi_{1} x+\xi_{2} y, x\right]+\left[z, a_{11} x+a_{12} y\right] \\
& =\left(a_{11}+a_{22}\right) y+a_{11} y .
\end{aligned}
$$

Hence $a_{11}=0$. This is the Hopf algebra described in Example 4.6.

4.6. Proof of the main result. With the help of the last few subsections, we are able to deliver the main theorem of the paper.

Theorem 4.24 (Theorem 0.3). Let $H$ be a connected Hopf algebra of GK-dimension 4 over an algebraically closed field of characteristic zero. Then $H$ is isomorphic to one of the following:

(a) Enveloping algebra $U(\mathfrak{g})$ over a Lie algebra $\mathfrak{g}$ of dimension 4 . Note that all 4-dimensional Lie algebras over the complex numbers $\mathbb{C}$ are listed in the book OV, Theorem 1.1(iv), page 209].

(b) Enveloping algebra $U(L)$ over an anti-cocommutative $C L A L$ of dimension 4. All anti-cocommutative coassociative Lie algebras of dimension 4 are classified in Theorem 3.5 .

(c) Primitively-thin Hopf algebras of GK-dimension 4. All primitively-thin Hopf algebras of GK-dimension 4 are classified in Theorem 4.23 .

Proof. By Lemma 1.3(e), $p(H) \geq 2$. Since $U(\mathfrak{g})$ embeds in $H$ where $\mathfrak{g}=P(H)$, we have $p(H) \leq 4$.

If $p(H)=4$, by Theorem 2.7 $H \cong U(\mathfrak{g})$ where $\mathfrak{g}=P(H)=P_{2}(H)$. This is case (a).

If $p(H)=3$, by Theorem 2.7. $H$ is isomorphic to the enveloping algebra $U(L)$ over an anti-cocommutative CLA $L$ of dimension 4. Anti-cocommutative CLAs of dimension 4 are classified in Theorem 3.5 . 
If $p(H)=2$, by definition, $H$ is a primitively-thin Hopf algebra of GK-dimension 4 , as classified in Theorem 4.23 ,

Corollary 4.25. Let $H$ be a connected Hopf algebra of dimension at most 4. Then, as an algebra, $H$ is isomorphic to $U(\mathfrak{g})$ for some Lie algebra $\mathfrak{g}$.

Proof. This is clear if GKdim $H \leq 3$. For GKdim $H=4$, the assertion is clear for the case when $p(H) \geq 3$. The only case left is when $p(H)=2$, in which the assertion was checked case-by-case in Examples 4.4 4.7.

\section{ACKNOWLEDGMENTS}

The authors thank Nicolás Andruskiewitsch and Ken Brown for their valuable suggestions and several conversations during the Banff workshop in October 2012 and the referee for a careful reading and useful comments. The first author was supported by the National Natural Science Foundation of China (No. 11471186) and the Shandong Provincial Natural Science Foundation of China (No. ZR2011AM013). The second and third authors were supported by the US National Science Foundation (NSF grant No. DMS 0855743).

\section{REFERENCES}

[AA] Nicolás Andruskiewitsch and Iván Ezequiel Angiono, On Nichols algebras with generic braiding, Modules and comodules, Trends Math., Birkhäuser Verlag, Basel, 2008, pp. 4764, DOI 10.1007/978-3-7643-8742-6_3. MR2742620 (2012b:16079)

[AS1] Nicolás Andruskiewitsch and Hans-Jürgen Schneider, Pointed Hopf algebras, New directions in Hopf algebras, Math. Sci. Res. Inst. Publ., vol. 43, Cambridge Univ. Press, Cambridge, 2002, pp. 1-68. MR1913436 (2003e:16043)

[AS2] Nicolás Andruskiewitsch and Hans-Jürgen Schneider, A characterization of quantum groups, J. Reine Angew. Math. 577 (2004), 81-104, DOI 10.1515/crll.2004.2004.577.81. MR2108213 (2005i:16083)

[Be] George M. Bergman, The diamond lemma for ring theory, Adv. in Math. 29 (1978), no. 2, 178-218, DOI 10.1016/0001-8708(78)90010-5. MR.506890(81b:16001)

[Br1] Kenneth A. Brown, Noetherian Hopf algebras, Turkish J. Math. 31 (2007), no. suppl., 7-23. MR2368080 (2008j:16106)

[Br2] Kenneth A. Brown, Representation theory of Noetherian Hopf algebras satisfying a polynomial identity (Seattle, WA, 1997), Contemp. Math., vol. 229, Amer. Math. Soc., Providence, RI, 1998, pp. 49-79, DOI 10.1090/conm/229/03310. MR1676211 (99m:16056)

[Br3] Kenneth A. Brown, Noncommutative unipotent groups in characteristic 0, a lecture at the BIRS workshop on "New Trends in Noncommutative Algebra and Algebraic Geometry" from Oct. 28 to Nov. 2, 2012; video available at http://www.birs.ca/events/2012/5day-workshops/12w5049/videos/watch/201210301535-Brown.mp4

[BG1] K. A. Brown and K. R. Goodearl, Homological aspects of Noetherian PI Hopf algebras of irreducible modules and maximal dimension, J. Algebra 198 (1997), no. 1, 240-265, DOI 10.1006/jabr.1997.7109. MR.1482982 (99c:16036)

[BG2] Ken A. Brown and Ken R. Goodearl, Lectures on algebraic quantum groups, Advanced Courses in Mathematics. CRM Barcelona, Birkhäuser Verlag, Basel, 2002. MR 1898492 (2003f:16067)

[BZ] K. A. Brown and J. J. Zhang, Prime regular Hopf algebras of GK-dimension one, Proc. Lond. Math. Soc. (3) 101 (2010), no. 1, 260-302, DOI 10.1112/plms/pdp060. MR2661247(2011j:16063)

[GZ1] K. R. Goodearl and J. J. Zhang, Homological properties of quantized coordinate rings of semisimple groups, Proc. Lond. Math. Soc. (3) 94 (2007), no. 3, 647-671, DOI 10.1112/plms/pdl022. MR2325315 (2008e:20077)

[GZ2] K. R. Goodearl and J. J. Zhang, Noetherian Hopf algebra domains of GelfandKirillov dimension two, J. Algebra $\mathbf{3 2 4}$ (2010), no. 11, 3131-3168, DOI 10.1016/j.jalgebra.2009.11.001. MR.2732991 (2012d:16098) 
[Gr] Mikhael Gromov, Groups of polynomial growth and expanding maps, Inst. Hautes Études Sci. Publ. Math. 53 (1981), 53-73. MR623534 (83b:53041)

[Kh] V. K. Kharchenko, A quantum analogue of the Poincaré-Birkhoff-Witt theorem (Russian, with Russian summary), Algebra Log. 38 (1999), no. 4, 476-507, 509, DOI 10.1007/BF02671731; English transl., Algebra and Logic 38 (1999), no. 4, 259-276. MR1763385 (2001f:16075)

[KL] Günter R. Krause and Thomas H. Lenagan, Growth of algebras and Gelfand-Kirillov dimension, Revised edition, Graduate Studies in Mathematics, vol. 22, American Mathematical Society, Providence, RI, 2000. MR.1721834 (2000j:16035)

[Li] Gongxiang Liu, On Noetherian affine prime regular Hopf algebras of Gelfand-Kirillov dimension 1, Proc. Amer. Math. Soc. 137 (2009), no. 3, 777-785, DOI 10.1090/S00029939-08-09034-5. MR2457414 (2010c:16037)

[LWZ] D.-M. Lu, Q.-S. Wu, and J. J. Zhang, Homological integral of Hopf algebras, Trans. Amer. Math. Soc. 359 (2007), no. 10, 4945-4975, DOI 10.1090/S0002-9947-07-04159-1. MR2320655 (2008f:16083)

[LPWZ1] D.-M. Lu, J. H. Palmieri, Q.-S. Wu, and J. J. Zhang, A-infinity structure on Ext-algebras, J. Pure Appl. Algebra 213 (2009), no. 11, 2017-2037, DOI 10.1016/j.jpaa.2009.02.006. MR2533303 (2010e:16015)

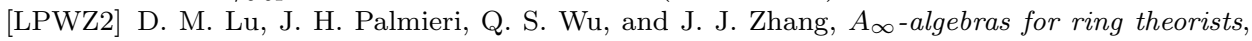
Proceedings of the International Conference on Algebra, Algebra Colloq. 11 (2004), no. 1, 91-128. MR2058967 (2005g:16015)

[MR] J. C. McConnell and J. C. Robson, Noncommutative Noetherian rings, with the cooperation of L. W. Small; A Wiley-Interscience Publication, Pure and Applied Mathematics (New York), John Wiley \& Sons Ltd., Chichester, 1987. MR.934572 (89j:16023)

[Mo] Susan Montgomery, Hopf algebras and their actions on rings, CBMS Regional Conference Series in Mathematics, vol. 82, Published for the Conference Board of the Mathematical Sciences, Washington, DC, by the Amer. Math. Soc., Providence, RI, 1993. MR.1243637 (94i:16019)

[OV] Lie groups and Lie algebras, III, Structure of Lie groups and Lie algebras; A translation of Current problems in mathematics. Fundamental directions. Vol. 41 (Russian), Akad. Nauk SSSR, Vsesoyuz. Inst. Nauchn. i Tekhn. Inform., Moscow, 1990 [ MR1056485 (91b:22001)]; Translation by V. Minachin [V. V. Minakhin]; Translation edited by A. L. Onishchik and È. B. Vinberg, Encyclopaedia of Mathematical Sciences, Vol. 41, Springer-Verlag, Berlin, 1994. MR1349140 (96d:22001)

[RZ] D. Rogalski and J. J. Zhang, Regular algebras of dimension 4 with 3 generators, New trends in noncommutative algebra, Contemp. Math., vol. 562, Amer. Math. Soc., Providence, RI, 2012, pp. 221-241, DOI 10.1090/conm/562/11139. MR2905562

[SSW] L. W. Small, J. T. Stafford, and R. B. Warfield Jr., Affine algebras of Gel'fand-Kirillov dimension one are PI, Math. Proc. Cambridge Philos. Soc. 97 (1985), no. 3, 407-414, DOI 10.1017/S0305004100062976. MR778674 (86g:16025)

[WZZ1] D.-G. Wang, J. J. Zhang, and G. Zhuang, Lower bounds of growth of Hopf algebras, Trans. Amer. Math. Soc. 365 (2013), no. 9, 4963-4986, DOI 10.1090/S0002-9947-201305793-5. MR3066776

[WZZ2] D.-G. Wang, J. J. Zhang, and G. Zhuang, Hopf algebras of GK-dimension two with vanishing Ext-group, J. Algebra 388 (2013), 219-247, DOI 10.1016/j.jalgebra.2013.03.032. MR3061686

[WZZ3] D.-G. Wang, J. J. Zhang, and G. Zhuang, Coassociative Lie algebras, Glasg. Math. J. 55 (2013), no. A, 195-215, DOI 10.1017/S001708951300058X. MR3110812

[WZZ4] D.-G. Wang, J. J. Zhang, and G. Zhuang, Primitive cohomology of Hopf algebras, arXiv 1411.4672 .

[We] Charles A. Weibel, An introduction to homological algebra, Cambridge Studies in Advanced Mathematics, vol. 38, Cambridge University Press, Cambridge, 1994. MR.1269324 (95f:18001)

[WuZ1] Q.-S. Wu and J. J. Zhang, Noetherian PI Hopf algebras are Gorenstein, Trans. Amer. Math. Soc. 355 (2003), no. 3, 1043-1066 (electronic), DOI 10.1090/S0002-9947-0203106-9. MR:1938745 (2003m:16056)

[WuZ2] Q.-S. Wu and J. J. Zhang, Regularity of involutory PI Hopf algebras, J. Algebra 256 (2002), no. 2, 599-610, DOI 10.1016/S0021-8693(02)00142-4. MR1939124(2004g:16042) 
[Zh1] Guangbin Zhuang, Existence of Hopf subalgebras of GK-dimension two, J. Pure Appl. Algebra 215 (2011), no. 12, 2912-2922, DOI 10.1016/j.jpaa.2011.04.012. MR 2811574 (2012k:16090)

[Zh2] Guangbin Zhuang, Properties of pointed and connected Hopf algebras of finite Gelfand-Kirillov dimension, J. Lond. Math. Soc. (2) 87 (2013), no. 3, 877-898, DOI 10.1112/jlms/jds079. MR3073681

School of Mathematical Sciences, Qufu Normal University, Qufu, Shandong 273165, People's Republic of ChinA

E-mail address: dgwang@mail.qfnu.edu.cn

E-mail address: dingguo95@126.com

Department of Mathematics, Box 354350, University of Washington, Seattle, WashINGTON 98195

E-mail address: zhang@math.washington.edu

Department of Mathematics, Box 354350, University of Washington, Seattle, WashINGTON 98195

E-mail address: gzhuang@math.washington.edu

Current address: Department of Mathematics, University of Southern California, 3620 S. Vermont Avenue, Los Angeles, California 90089-2532

E-mail address: gzhuang@usc.edu 